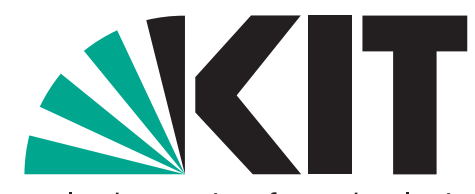

Karlsruher Institut für Technologie

\title{
On discounting and voting in a simple growth model
}

by Kirill Borissov, Mikhail Pakhnin, Clemens Puppe

No. 77 | DECEMBER 2015

\section{WORKING PAPER SERIES IN ECONOMICS}

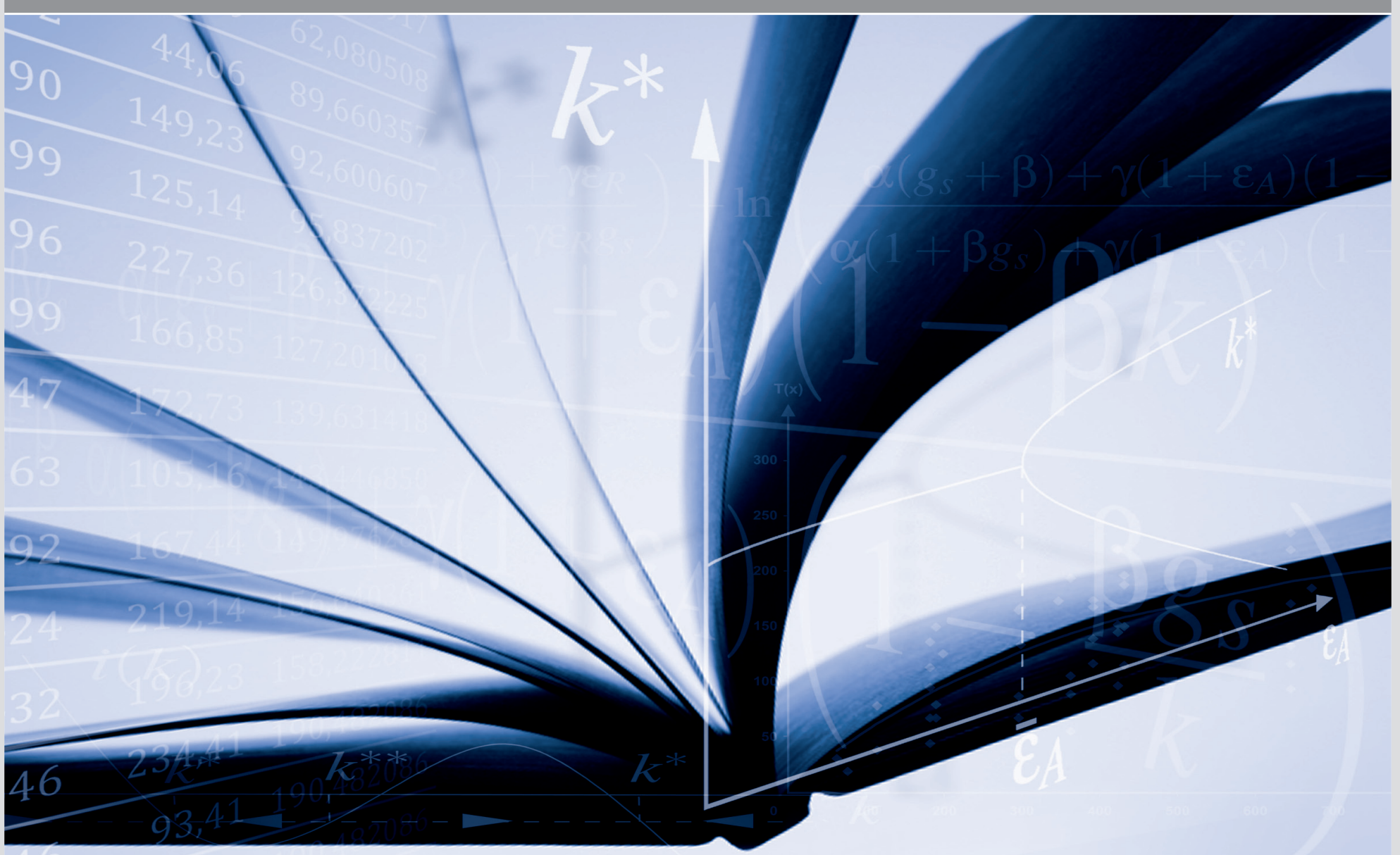




\section{Impressum}

Karlsruher Institut für Technologie (KIT)

Fakultät für Wirtschaftswissenschaften

Institut für Volkswirtschaftslehre (ECON)

Schlossbezirk 12

76131 Karlsruhe

KIT - Die Forschungsuniversität in der Helmholtz-Gemeinschaft

Working Paper Series in Economics

No. 77, December 2015

ISSN 2190-9806

econpapers.wiwi.kit.edu 


\title{
On Discounting and Voting in a Simple Growth Model*
}

\author{
Kirill Borissov† Mikhail Pakhnin; Clemens Puppe ${ }^{\S}$
}

December 2015

\begin{abstract}
In dynamic resource allocation models, the non-existence of voting equilibria is a generic phenomenon due to the multi-dimensionality of the choice space even with agents heterogeneous only in their discount factors. Nevertheless, at each point of time there may exist a "median voter" whose preferred instantaneous consumption rate is supported by a majority of agents. Based on this observation, we propose an institutional setup ("intertemporal majority voting") in a Ramsey-type growth model with common consumption and heterogeneous agents, and show that it provides a microfoundation of the choice of the optimal consumption stream of the median agent. While the corresponding intertemporal consumption stream is in general not a Condorcet winner among all feasible paths, its induced instantaneous consumption rate receives a majority at each point in time in the proposed intertemporal majority voting procedure. We also provide a characterization of balanced-growth and steadystate voting equilibria in the case in which agents may differ not only in their time preference, but also in their instantaneous utility functions.
\end{abstract}

JEL Classification: D11, D71, D91, O13, O43.

Keywords: collective choice, common-pool resource, economic growth, heterogeneous agents, median voter theorem.

\section{Introduction}

It is well-known that in multi-dimensional models a voting equilibrium under majority rule fails to exist generically (Plott, 1967; McKelvey, 1976). This holds in particular in dynamic multi-period resource allocation problems - even if choices at different points in time are linked by an intertemporal resource constraint (Boylan et al., 1996), and even

${ }^{*}$ We are grateful to the participants of the Fourth International Workshop on Natural Resources, Environment and Economic Growth St. Petersburg, Oct. 1-2, 2015, for their helpful comments and discussions. We also want to thank the participants of research seminars at Karlsruhe Institute of Technology and Paris West University Nanterre La Defense for their feedback on earlier versions of this paper.

${ }^{\dagger}$ European University at St. Petersburg, 3 Gagarinskaya St., St. Petersburg 191187, Russia, and St. Petersburg Institute for Economics and Mathematics (RAS), 36-38 Serpukhovskaya St., St. Petersburg 190013, Russia. E-mail: kirill@eu.spb.ru.

${ }^{\ddagger}$ European University at St. Petersburg, 3 Gagarinskaya St., St. Petersburg 191187, Russia, and St.Petersburg Institute for Economics and Mathematics (RAS), 36-38 Serpukhovskaya St., St. Petersburg 190013, Russia. E-mail: mpakhnin@eu.spb.ru.

$\S$ Karlsruhe Institute of Technology, Department of Economics and Management, Kaiserstrasse 12, 76128 Karlsruhe, Germany. E-mail: clemens.puppe@kit.edu. 
in cases where in addition the agents' type space is one-dimensional (see, e.g., De Donder et al., 2012). One-dimensional type spaces arise naturally when agents differ only in discount rates, a case that has received considerable attention recently (see, e.g., Heal and Millner, 2014; Jackson and Yariv, 2015).

Notwithstanding these negative results, at each point of time there may exist a "median voter" whose preferred choice of instantaneous consumption rate is supported by a majority of agents. Based on this observation, we propose a simple institutional setup in a Ramsey-type growth model, intertemporal majority voting, that does not suffer from the problem of generic non-existence of equilibrium. Importantly, in this setup the temporary voting is not over consumption levels but over consumption rates, since only the latter variable entails a degree of freedom given the intertemporal resource constraint and the decisions (on consumption rates) in all other periods. The equilibrium concept that we employ is Kramer-Shepsle equilibrium with perfect foresight; that is, (i) each period's decision follows the (sincere) majority vote under the assumption that agents maximize their utility given the outcomes of all other periods, and (ii) agents' expectations about these outcomes are correct in equilibrium ("perfect foresight"). It is worth emphasizing that the institutional framework is well-defined also without the assumptions of utility maximization and/or perfect foresight; evidently, in particular the latter condition is quite restrictive.

Our main result shows that there is a unique Kramer-Shepsle equilibrium which yields as outcome the optimal consumption stream of the agent with the median discount factor. As an important intermediate step we establish that, for each fixed agent, the step-by-step determination of the optimal consumption rate under perfect foresight yields the optimal overall intertemporal consumption stream. While this technical result probably belongs to the body of "folk wisdom" within the Ramsey model, we are not aware of a rigorous proof and provide one here.

In our basic model, we assume that agents have the same instantaneous utility function and differ only in their discount rates. We also consider the case of agents with different utility functions. For this general case we provide a characterization of balanced-growth and steady-state voting equilibria.

\subsection{Common property resource problems}

The problem of aggregating heterogeneous time preferences arises in many contexts. ${ }^{1}$ It is instructive to consider this problem within the common property resource framework both with renewable and exhaustible resources. Examples are the hunting for animals, the grazing of cattle on a common ground, the pollution of the atmosphere, or the drilling for oil in a common underground reservoir.

In these contexts, an issue of evident importance is the determination of the socially desirable harvest rate. Concretely, consider a village situated near a fishing ground. The fishing ground is self-managed by village citizens, who differ in their time preferences. The question is: what is the harvest rate of the fish stock collectively set by heterogeneous agents? If all citizens in the village were identical, then the rate of the fish stock exploitation could be easily determined using their common discount factor. However, it is not at all clear how to determine the harvest rate when people have different discount factors.

\footnotetext{
${ }^{1}$ For recent evidence that individuals indeed differ in their discount factors see, e.g., Wang et al. (2010), Castillo et al. (2011), and Schaner (2015).
} 
One might try to argue that the introduction of property rights can (indirectly) solve the problem. Indeed, the typical and well-known solution to the "tragedy of the commons" is to establish private property rights. Once the property rights are enforced, each owner just acts optimally according to her own time preference. This might indeed circumvent the problem in cases where suitable property rights can be established. ${ }^{2}$

However, often the non-excludability of common goods prevents the enforcement of suitable private property rights. This is likely to occur in the case of the underground oil reservoir, the fishing ground, or the so-called "global commons". For instance, the tendency of fish to migrate makes it impossible to define geographically determined property rights over the fish stock. In this case a solution may be to introduce a governmental or community resource ownership, but then it is necessary to find a non-market mechanism of determining the harvest rate.

\subsection{Social choice in the optimal growth model}

A natural framework for the analysis of collective intertemporal decision problems of the sort just described is the optimal growth model originally developed by Ramsey (1928). This model can be generalized to a multi-agent model with several types of individuals differentiated by their rates of time preference. There is a vast amount of literature devoted to the Ramsey general equilibrium models with heterogeneous agents who differ in their discount factors (see Becker, 2006, for an excellent survey). In this kind of models each agent separately solves her own optimization problem and thus has an independent private consumption stream.

However, the Ramsey framework also allows one to study how heterogeneous agents make joint decisions over common consumption streams. Note that it does not matter whether "common consumption" is a collectively consumed public good or a private good that is consumed according to some fixed and commonly known sharing rule. What is important is that agents' personal utilities are based on their collective decisions, i.e., on the common consumption stream they choose. Here, economic growth theory meets social choice theory, and there is indeed a literature that analyzes how political institutions can be incorporated into growth models in order to determine collective choices among heterogeneous agents (see, e.g., Beck, 1978; Boylan, 1995; Boylan et al., 1996).

A natural way of aggregating heterogeneous preferences is voting. Suppose that agents vote over all feasible consumption streams by pairwise majority voting. Then, it is wellknown that, due to the high dimensionality of the underlying choice space, there does in general not exist a Condorcet winner, i.e., for every feasible consumption stream there exists another feasible consumption stream that is preferred by a majority (see, e.g., Davis et al., 1972; Kramer, 1973; Bucovetsky, 1990). ${ }^{3}$ Moreover, the fact that agents differ only in one parameter does not help: there still is no Condorcet winner in voting over multi-dimensional choice space even if the agents' type space is one-dimensional (see, e.g., De Donder et al., 2012). Boylan et al. (1996) consider voting over feasible consumption paths in the Ramsey optimal growth model and prove that there is in general no Condorcet winner. In a more recent paper, Jackson and Yariv (2015) analyze the case of agents who differ only in their discount factor, and prove a general impossibility theorem which implies, among other things, that any non-dictatorial aggregation rule of feasible

\footnotetext{
${ }^{2}$ If the "owners" are groups of individuals with heterogeneous time preferences, the problem might of course persist within these groups.

${ }^{3}$ Bernheim and Slavov (2007) characterized this kind of situation as the "curse of dimensionality".
} 
consumption plans satisfying mild further conditions admits cyclical social preferences.

Despite these negative results, it appears that in a model in which agents differ only in their discount factor, the optimal consumption path of the agent with the median discount factor has some claim for being a natural and appealing collective choice. But clearly, the mentioned impossibility results imply in particular that also the optimal consumption path for the median agent cannot be a Condorcet winner among all feasible paths, i.e., there generally exist feasible consumption paths that are preferred by a majority of agents to the optimal consumption path of the median agent. ${ }^{4}$

One way to overcome this difficulty has been considered by Beck (1978) and, more recently, by Heal and Millner (2014). In these models, agents are simply only allowed to vote over the set of individually optimal paths. It can be shown that, among all individually optimal paths, the optimal path for the agent with the median discount factor is indeed a Condorcet winner. However, ensuring the existence of a stable voting outcome in this way is not very satisfactory since it is made possible only by severely restricting the choice set.

A different voting mechanism in the Ramsey framework is proposed by Boylan et al. (1996). These authors introduce two additional agents ("political candidates") to the model. In each period the candidates propose a consumption level for the agents and care only about being elected. Agents vote for one of the candidates and care only about consumption. A specific noncooperative game is then constructed, and it is shown that the subgame perfect equilibrium coincides with the optimal path for the median agent. Although it yields the desired and intuitive outcome, this voting procedure seems quite contrived and complex.

The purpose of the present paper is to propose a more intuitive and tractable voting procedure that yields the optimal consumption path of the agent with the median discount factor as outcome. ${ }^{5}$

\subsection{Intertemporal majority voting over consumption rates}

We consider a Ramsey-type growth model with agents differing in their time preferences. Agents maximize their intertemporal discounted utilities by allocating at each point in time a given amount of single good between consumption, which provides instant utility, and investment, which is used in production. The technology is described by a neoclassical production function.

We suppose that agents share a common consumption stream. The good is either consumed collectively, or privately according to some fixed sharing rule. In the common property resource interpretation of the Ramsey model with common consumption, the capital stock is viewed as the resource stock, and the consumption level is the amount

\footnotetext{
${ }^{4}$ In fact, one can show that there exist feasible paths that are preferred to the optimal path of the median agent by all other agents. These paths are obtained by increasing the consumption in earlier and later periods, which makes both the impatient and the patient agents better off, and by reducing the consumption in intermediate periods in order to make the path feasible.

${ }^{5}$ The general idea to use dynamic voting in order to determine a stable outcome has been investigated in a number of specific models. In Borissov et al. (2014a) agents vote for a tax aimed at environmental maintenance; Borissov et al. (2014b) study voting over the shares of public goods in GDP, and Borissov and Pakhnin (2014) consider voting over extraction rates in two models of economic growth with exhaustible natural resources. In all cases, the equilibrium policy is determined by the agent with the median discount factor as in the present paper. However, these models lack generality because they use specific forms of the utility and production functions.
} 
of the resource extracted. In other words, the consumption level equals the harvest rate (extraction rate in the exhaustible resource case) times the available resource stock. The production function becomes the regeneration function if the resource is renewable, and represents the pure storage technology if the resource is exhaustible.

Within this framework, we propose a simple and natural voting mechanism according to which agents choose a consumption path for society from the set of all feasible consumption paths by "intertemporal majority voting." The two crucial principles in this institutional setup are that (i) voting is done "step-by-step" at each point of time, and (ii) the vote is not over the consumption level itself, but over the consumption rate. We thus avoid the "curse of dimensionality" by converting a multi-dimensional choice space into a series of one-dimensional choice spaces. Indeed, the dynamic intertemporal structure of the Ramsey model naturally suggests to consider institutions that also allow for intertemporal choices of agents. The solution concept given the proposed intertemporal voting procedure is Kramer-Shepsle equilibrium (Kramer, 1972; Shepsle, 1979). In general, a vector of policies is a Kramer-Shepsle equilibrium if, for any single dimension, the corresponding policy in this dimension coincides with the majority choice, given the equilibrium choices in all other dimensions. ${ }^{6}$

Given the general idea to transform the multi-dimensional choice problem into a sequence of one-dimensional choice problems, an important issue that has to be addressed is what the choice variable should be. Indeed, one-dimensional voting over the current consumption level given the consumption levels in all other periods is pointless, since consumption in each period is uniquely determined by consumption in all other periods via the overall resource constraint. Thus, there is no trade-off between consumption today and consumption in the future (we provide a simple example in Section 3 below to illustrate this point), and formally, every feasible consumption path is a Kramer-Shepsle equilibrium in the Ramsey model.

To implement the idea of intertemporal majority voting in a fruitful manner, we have to look at the Ramsey model from a slightly different perspective. Originally, the model is formulated in terms of consumption levels, and mathematically, it involves an optimal control problem with the consumption level as the control variable. We make a change of variables and use instead the consumption rate (i.e., 1 - savings rate) as control variable. ${ }^{7}$ Using this change of variable, we define a voting equilibrium in two stages. First, for any point in time agents vote by majority rule over the current consumption rate, given the current capital stock and their expectations about future consumption rates. This yields a one-dimensional decision problem, and we show that agents' preferences over the current consumption rate are single-peaked. If, in addition, agents have the same instantaneous utility function and the same expectations, at each given point in time the temporary voting equilibrium (i.e., the instantaneous Condorcet winner) is the preferred consumption rate of the agent with the median discount factor. The intertemporal majority voting equilibrium is then defined as a sequence of temporary voting outcomes such that all agents' expectations are correct. We prove that if agents have the same instantaneous utility function, there is a unique intertemporal voting equilibrium, which is given by the

\footnotetext{
${ }^{6}$ Clearly, if the multi-dimensional problem admits a Condorcet winner, then the Condorcet winner constitutes a Kramer-Shepsle equilibrium.

${ }^{7}$ The savings rate as control variable in the Ramsey model has been used by Phelps and Pollak (1968) and Peleg and Yaari (1973). These authors study agents' behavior in the optimal growth model with time-inconsistent preferences, and ask under which conditions the chosen plan of actions will be actually followed by rational individuals in the future. By contrast, we assume time-consistent preferences throughout.
} 
optimal consumption path for the agent with the median discount factor. The proof is based on the general result that the step-by-step determination of the consumption rate under perfect foresight for any given agent results in the ("once-and-for-all") optimum in terms of consumption levels for this agent. This result, though not surprising, is of interest in itself, and we discuss it in some detail in Section 4 below.

We thus view our analysis as providing an institutional "microfoundation" for the choice of the optimal consumption path of the agent with the median discount factor, a proposal that has been repeatedly put forward in the literature but without an ultimately appealing justification so far. If agents differ both in their discount factors and instantaneous utility functions, the intertemporal voting equilibrium is clearly no longer determined by the discount factor alone. However, we are still able to generalize our analysis to two special cases of such multi-dimensional heterogeneity.

The first is the case when the production function is linear and the utility functions of agents have constant elasticities of intertemporal substitution. We define a balancedgrowth voting equilibrium, prove its uniqueness and show that it only depends on the median growth rate. The second is the case when the production function is strictly concave, while utility functions are completely general. In this case, we define a steadystate voting equilibrium, and show that it is unique and fully determined by the agent with the median discount factor.

\subsection{Further remarks on related literature}

The outcome of our notion of intertemporal majority voting (i.e., the optimal consumption path of the median agent) is clearly both time-consistent and Pareto efficient. This may seem at odds with the result of Jackson and Yariv (2015), who argue that any time-consistent and Pareto efficient voting rule must be dictatorial. There is in fact no contradiction here since the choice of an optimal path of one agent is indeed ex post "dictatorial." Note, however, that unlike Jackson's and Yariv's notion, the usual definition of dictatorship in social choice theory is a much stronger notion of ex ante dictatorship: an aggregation rule is dictatorial if only one agent is decisive no matter what the preferences of all other agents' are. Evidently, any aggregation rule that follows the preferences of the median voter is necessarily "ex post dictatorial," but this does not imply dictatorship in the usual sense of social choice theory. The same holds for the choice of the optimal consumption path of the median agent under our institutional setup.

We emphasize that in this paper we do not address questions related to uncertainty of future economic development. There is a lively and ongoing discussion on how to discount the future under uncertainty (see, e.g., Pearce et al., 2003; Gollier and Weitzman, 2010; Traeger, 2013). While the introduction of uncertainty seems to bring the problem of choosing a consumption path closer to real life decisions, it also complicates matters quite dramatically. Our hope is that, even though our analysis does not directly contribute to the literature of discounting the future under uncertainty, the idea of intertemporal majority voting might be also fruitfully applicable to this more general setting.

The rest of the paper is organized as follows. Section 2 reviews the basic setup of the Ramsey model in terms of consumption levels. Section 3 provides a simple but instructive example of implementing the step-by-step optimization and voting procedures. In Section 4 we present the Ramsey model in terms of consumption rates and consider the stepby-step decision-making process. We define a step-by-step intertemporal optimum and 
show that in the Ramsey model a step-by-step intertemporal optimum coincides with the optimum in terms of consumption rates. In Section 5 we proceed to intertemporal majority voting over consumption rates and define an intertemporal voting equilibrium. Section 6 states our main result under the assumption that all agents have the same instantaneous utility function. In this case an intertemporal voting equilibrium is unique and coincides with the optimum in terms of consumption rates for the agent with the median discount factor. In Section 7 we study the general case where agents differ both in their discount factors and utility functions. Section 8 concludes.

\section{The model}

We consider a Ramsey-type growth model with heterogeneous agents and common consumption. Suppose $T \in \mathbb{N} \cup\{\infty\}$ is the length of the time horizon, which can be finite or infinite. Let time be $\mathbb{T}=\{0,1, \ldots, T\}$ when $T<\infty$, and $\mathbb{T}=\{0,1, \ldots\}$ when $T=\infty$.

There is an odd number $N$ of heterogeneous agents indexed by $i=\{1,2, \ldots, N\}$. The heterogeneity is captured by agents' discount factors and utility functions. Suppose agent $i$ has a discount factor $\delta_{i} \in(0,1)$, and an instantaneous utility function $u_{i}(c)$ which satisfies the following conditions:

$$
u_{i}(c): \mathbb{R}_{++} \rightarrow \mathbb{R}, \quad u_{i}^{\prime}(c)>0, \quad u_{i}^{\prime \prime}(c)<0, \quad \lim _{c \rightarrow 0} u_{i}^{\prime}(c)=+\infty .
$$

Agent's preferences over consumption streams $C=\left\{c_{t}\right\}_{t \in \mathbb{T}}$ are represented by the intertemporal utility function

$$
\mathcal{U}^{i}=\sum_{t \in \mathbb{T}} \delta_{i}^{t} u_{i}\left(c_{t}\right)
$$

We consider the economy in which a single homogeneous good is produced. In each period $t \in \mathbb{T}$ the available amount of good in the economy is allocated between consumption ${ }^{8}$ $c_{t}$ and capital $k_{t+1}$ for use in the next period production:

$$
c_{t}+k_{t+1}=f\left(k_{t}\right), \quad t \in \mathbb{T},
$$

where $f(k)$ is the production function. We assume that $f(k)$ either is linear,

$$
f(k)=A k, \quad A>0,
$$

or satisfies the following properties:

$$
f(0)=0, \quad f^{\prime}(k)>0, \quad f^{\prime \prime}(k)<0, \quad \exists \bar{k}: f(\bar{k})=\bar{k}, \quad \delta_{\min } f^{\prime}(0)>1,
$$

where $\delta_{\min }$ is the minimal discount factor in the set $\left\{\delta_{i}\right\}_{i=1}^{N} \cdot{ }^{9}$

Suppose $k_{0}>0$ is given. We call a sequence $\{C, K\}=\left\{c_{t}, k_{t+1}\right\}_{t \in \mathbb{T}}$ a feasible path if

$$
c_{t}+k_{t+1}=f\left(k_{t}\right), \quad c_{t} \geq 0, \quad k_{t+1} \geq 0, \quad t \in \mathbb{T} .
$$

\footnotetext{
${ }^{8}$ We suppose that the good is either consumed collectively or it is consumed privately, in which case there is some fixed and commonly known sharing rule. If, for instance, this rule is egalitarian, then $u_{i}(c)$ should be replaced with $u_{i}(c / N)$. However, this is of no importance for our considerations and results. ${ }^{9}$ In the common property resource problem interpretation, $f(k)=k$ describes the exhaustible resource stock dynamics, and the Ramsey problem becomes the "cake-eating" problem of Gale (1967). A function $f(k)$ that satisfies $(3)$ describes a regenerative capacity of the renewable resource.
} 
Consider the following optimization problem:

$$
\begin{gathered}
\max \sum_{t \in \mathbb{T}} \delta_{i}^{t} u_{i}\left(c_{t}\right), \\
\text { s. t. } \quad c_{t}+k_{t+1}=f\left(k_{t}\right), \quad c_{t} \geq 0, \quad k_{t+1} \geq 0, \quad t \in \mathbb{T} .
\end{gathered}
$$

Definition. A solution to problem (4), $\left\{C^{i *}, K^{i *}\right\}=\left\{c_{t}^{i *}, k_{t+1}^{i *}\right\}_{t \in \mathbb{T}}$, is called an optimum in terms of consumption levels for agent $i$.

If the production functions satisfies (3), then maximization problem (4) has a unique solution (optimal path). If the production functions satisfies (2), then in what follows we additionally assume that problem (4) has a solution (optimal path) for each agent $i$.

\section{Intertemporal majority voting: an example}

The most natural idea to overcome the absence of a Condorcet winner is to convert a multi-dimensional choice space, made up of sequences of consumptions, into a series of one-dimensional choice spaces. The basic idea of "coordinate-wise" majority voting was proposed independently by Kramer (1972) and Shepsle (1979).

Suppose that today agents vote only over current consumption, under some expectations about future consumption. We may hope that there exists a stable outcome of this one-dimensional voting. However, this reasonable and appealing idea cannot be applied directly to voting over consumption streams. To see why, consider the following three-period example.

Suppose the intertemporal utility functions in terms of consumption levels are loglinear. Suppose also the production function is $f(k)=k$, so that the resource constraints are

$$
c_{0}+k_{1}=k_{0}, \quad c_{1}+k_{2}=k_{1}, \quad c_{2}+k_{3}=k_{2},
$$

where $k_{0}>0$ is given. It is evident that $k_{3}$ must be zero, so the overall resource constraint is given by

$$
c_{0}+c_{1}+c_{2}=k_{0} .
$$

The utility maximization problem in terms of consumption levels for agent $i$ is as follows:

$$
\begin{gathered}
\max \left\{\ln c_{0}+\delta_{i} \ln c_{1}+\delta_{i}^{2} \ln c_{2}\right\}, \\
\text { s. t. } \quad c_{0}+c_{1}+c_{2}=k_{0} .
\end{gathered}
$$

The solution to this problem is:

$$
\begin{gathered}
c_{0}^{i *}=\frac{k_{0}}{1+\delta_{i}+\delta_{i}^{2}}, \quad c_{1}^{i *}=\frac{\delta_{i} k_{0}}{1+\delta_{i}+\delta_{i}^{2}}, \quad c_{2}^{i *}=\frac{\delta_{i}^{2} k_{0}}{1+\delta_{i}+\delta_{i}^{2}}, \\
k_{1}^{i *}=\frac{\left(\delta_{i}+\delta_{i}^{2}\right) k_{0}}{1+\delta_{i}+\delta_{i}^{2}}, \quad k_{2}^{i *}=\frac{\delta_{i}^{2} k_{0}}{1+\delta_{i}+\delta_{i}^{2}}, \quad k_{3}^{i *}=0 .
\end{gathered}
$$

Suppose that at time 0 agents have some expectations about future consumption, $c_{1}$ and $c_{2}$. Agents vote over the time 0 consumption $c_{0}$. The preferred time 0 consumption for agent $i$ is a solution to the following problem:

$$
\begin{gathered}
\max _{c_{0}} \ln c_{0}, \\
\text { s. t. } \quad c_{0}=k_{0}-\left(c_{1}+c_{2}\right) .
\end{gathered}
$$


However, this optimization problem is degenerate. The overall resource constraint under given expectations determines a unique feasible and hence the optimal value of $c_{0}$. Moreover, this value is the same for all agents.

Thus if agents choose current consumption under some expectations about the future, then it is optimal for all agents to consume today as much as possible, given the future consumption profile and the initial amount of capital. It follows that every feasible path, i.e., the sequence $\left\{c_{0}, c_{1}, c_{2}\right\}$ such that $c_{0}+c_{1}+c_{2}=k_{0}$, can be a result of intertemporal majority voting under perfect foresight. This voting mechanism seems to be quite senseless.

However, this example does not reduce the power of the idea to convert voting over the multi-dimensional sequence into the sequence of the one-dimensional votes. To look at the same example from a different perspective, let us formulate the initial problem in terms of consumption rates,

$$
e_{t}=\frac{c_{t}}{f\left(k_{t}\right)}=\frac{c_{t}}{k_{t}}
$$

instead of consumption levels $c_{t}$. It is clear that $0 \leq e_{t} \leq 1$.

The objective function in terms of consumption rates of agent $i$ takes the form

$$
V^{i}\left(e_{0}, e_{1}, e_{2}\right)=\ln \left(e_{0} k_{0}\right)+\delta_{i} \ln \left(e_{1}\left(1-e_{0}\right) k_{0}\right)+\delta_{i}^{2} \ln \left(e_{2}\left(1-e_{1}\right)\left(1-e_{0}\right) k_{0}\right),
$$

where $k_{0}>0$ is given.

The solution to the utility maximization problem in terms of consumption rates (optimum in terms of consumption rates) for agent $i$ is:

$$
\left\{e_{0}^{i *}, e_{1}^{i *}, e_{2}^{i *}\right\}=\left\{\frac{1}{1+\delta_{i}+\delta_{i}^{2}}, \frac{1}{1+\delta_{i}}, 1\right\}
$$

Now let us apply the intertemporal majority voting procedure to the problem formulated in terms of consumption rates. Agents vote over the current consumption rate under given past consumption rates and expectations about future consumption rates. Suppose that at time 0 expectations about future consumption rates are $e_{1}$ and $e_{2}$. Then the preferred time 0 consumption rate for agent $i, e_{0}^{i}$, is the solution to the following problem:

$$
\max _{0 \leq e_{0} \leq 1} V^{i}\left(e_{0}, e_{1}, e_{2}\right)
$$

Since

$$
\frac{\partial V^{i}\left(e_{0}, e_{1}, e_{2}\right)}{\partial e_{0}}=0 \Leftrightarrow \frac{1}{e_{0}}=\frac{\delta_{i}}{\left(1-e_{0}\right)}+\frac{\delta_{i}^{2}}{\left(1-e_{0}\right)}
$$

we obtain $e_{0}^{i}=\frac{1}{1+\delta_{i}+\delta_{i}^{2}}$.

In this particular case, due to the simplicity of the example, $e_{0}^{i}$ does not depend on expectations about future consumption rates. Note also, that it coincides with $e_{0}^{i *}$, which is the first element in the optimum in terms of consumption rates for this agent.

It is easily seen that preferences of agents in one-dimensional voting over $e_{0}$ are singlepeaked, and the preferred values $e_{0}^{i}$ are decreasing in $\delta_{i}$. By the median voter theorem, a Condorcet winner is the preferred time 0 consumption rate for the median agent, $e_{0}^{*}=$ $\frac{1}{1+\delta_{\text {med }}+\delta_{\text {med }}^{2}}$, where $\delta_{\text {med }}$ is the median discount factor.

Now consider voting over the time 1 consumption rate. Agents already know the time 0 consumption rate, which is the winner $e_{0}^{*}$. They also have expectations about the time 
2 consumption rate, $e_{2}$. Then the preferred time 1 consumption rate for agent $i, e_{1}^{i}$, is a solution to the following problem:

$$
\max _{0 \leq e_{1} \leq 1} V^{i}\left(e_{0}^{*}, e_{1}, e_{2}\right)
$$

We have

Thus $e_{1}^{i}=\frac{1}{1+\delta_{i}}$.

$$
\frac{\partial V^{i}\left(e_{0}^{*}, e_{1}, e_{2}\right)}{\partial e_{1}}=0 \Leftrightarrow \frac{\delta_{i}}{e_{1}}=\frac{\delta_{i}^{2}}{\left(1-e_{1}\right)}
$$

It is clear that the preferred time 1 consumption rate for each agent does not depend on previous decisions or expectations about future consumption rates, and coincides with the second element in the optimum in terms of consumption rates for the corresponding agent. By the median voter theorem, a Condorcet winner is the preferred time 1 consumption rate for the median agent, $e_{1}^{*}=\frac{1}{1+\delta_{\text {med }}}$.

Finally, the problem of finding the preferred time 2 consumption rate for agent $i, e_{2}^{i}$, takes the form:

$$
\max _{0 \leq e_{2} \leq 1} V^{i}\left(e_{0}^{*}, e_{1}^{*}, e_{2}\right)
$$

Clearly, the solution is $e_{2}^{i}=e_{2}^{i *}=1$. Since all agents vote unanimously, a Condorcet winner is $e_{2}^{*}=1$.

Thus we obtain the sequence of consumption rates

$$
E^{*}=\left\{e_{0}^{*}, e_{1}^{*}, e_{2}^{*}\right\}=\left\{\frac{1}{1+\delta_{\text {med }}+\delta_{\text {med }}^{2}}, \frac{1}{1+\delta_{\text {med }}}, 1\right\} .
$$

Each element of $E^{*}$ is a Condorcet winner in one-dimensional voting over the single consumption rate at the corresponding moment of time under known values of previous consumption rates and under given expectations about future consumption rates.

It is easily seen that the sequence $E^{*}$ is the solution to the utility maximization problem in terms of consumption rates for the median agent. It is also clear that $E^{*}$ corresponds to the solution to the utility maximization problem in terms of consumption levels for the median agent.

This simple example illustrates two important aspects of using consumption rate as the control variable. First, for each agent step-by-step optimization under given values of past consumption rates and expectations about future consumption rates yields the same result as the optimum in terms of consumption rates. Second, intertemporal majority voting over consumption rates yields the optimum in terms of consumption rates for the median agent as the outcome.

There is a natural question: can the step-by-step optimizing procedure (and hence the intertemporal majority voting procedure) be generalized to any Ramsey-type model? In the general case, the outcome of the time $\tau$ optimization problem (i.e., the preferred time $\tau$ consumption rate) for each agent is a function of all future consumption rates. Hence to apply a step-by-step optimization procedure in the general case, we need to introduce and specify agents' expectations about future consumption rates.

If agents form naive expectations, there is no reason to presume that the outcome of the step-by-step optimization procedure is optimal. However, we show that if an agent has perfect foresight about future decisions, then the sequence of consumption rates chosen step by step coincides with her optimum in terms of consumption rates. Moreover, if agents' utility functions are identical, the outcome of the intertemporal majority voting procedure under perfect foresight is the optimum in terms of consumption rates for the agent with the median discount factor. 


\section{Step-by-step intertemporal optimum}

In this section we consider the optimization problem in terms of consumption rates, define a step-by-step intertemporal optimum and show that in the Ramsey model a step-by-step intertemporal optimum coincides with the optimum in terms of consumption rates. This simple result seems not surprising and is in fact quite natural. However, to the best of our knowledge, it has not yet been explicitly stated and proved.

In the optimal control problem (4) the control variable is consumption $c_{t}$. We make a change of variables:

$$
e_{t}=\frac{c_{t}}{f\left(k_{t}\right)}
$$

and rewrite the initial problem in terms of consumption rates. ${ }^{10}$ Since $k_{t+1} \geq 0$ and $c_{t} \geq 0$, it follows that $0 \leq e_{t} \leq 1$. We call a sequence $E=\left\{e_{t}\right\}_{t \in \mathbb{T}}$, such that $0 \leq e_{t} \leq 1$ for all $t \in \mathbb{T}$, a feasible sequence of consumption rates.

Taking into account (5) and the constraints in (4), we can express consumption and the capital stock at time $t$ in terms of $k_{0}$ and all previous consumption rates:

$$
\begin{aligned}
& c_{t}=e_{t} f\left(\left(1-e_{t-1}\right) f\left(\left(1-e_{t-2}\right) f\left(\ldots f\left(k_{0}\right)\right)\right)\right), \quad t \in \mathbb{T}, \\
& k_{t+1}=\left(1-e_{t}\right) f\left(\left(1-e_{t-1}\right) f\left(\left(1-e_{t-2}\right) f\left(\ldots f\left(k_{0}\right)\right)\right)\right), \quad t \in \mathbb{T} .
\end{aligned}
$$

It is clear that given $k_{0}$, a feasible path $\left\{c_{t}, k_{t+1}\right\}_{t \in \mathbb{T}}$ uniquely corresponds to a feasible sequence of consumption rates $\left\{e_{t}\right\}_{t \in \mathbb{T}}$, and vice versa.

Using (6), we incorporate the resource constraints into the objective function which takes the form:

$$
\begin{gathered}
\mathcal{V}^{i}\left(e_{0}, e_{1}, \ldots\right)=u_{i}\left(e_{0} f\left(k_{0}\right)\right)+ \\
\delta_{i} u_{i}\left(e_{1} f\left(\left(1-e_{0}\right) f\left(k_{0}\right)\right)\right)+\delta_{i}^{2} u_{i}\left(e_{2} f\left(\left(1-e_{1}\right) f\left(\left(1-e_{0}\right) f\left(k_{0}\right)\right)\right)\right)+\ldots
\end{gathered}
$$

Problem (4), rewritten in terms of consumption rates, is given by:

$$
\begin{gathered}
\max \sum_{t \in \mathbb{T}} \delta_{i}^{t} u_{i}\left(e_{t} f\left(\left(1-e_{t-1}\right) f\left(\left(1-e_{t-2}\right) f\left(\ldots f\left(k_{0}\right)\right)\right)\right)\right), \\
\text { s. t. } 0 \leq e_{t} \leq 1, \quad t \in \mathbb{T}
\end{gathered}
$$

Definition. A solution to problem (8), $E^{i *}=\left\{e_{t}^{i *}\right\}_{t \in \mathbb{T}}$, is called an optimum in terms of consumption rates for agent $i$.

Suppose $k_{0}>0$ is given. It is clear that if $\left\{C^{i *}, K^{i *}\right\}$ is the optimum in terms of consumption levels, then the sequence $E^{i *}$ constructed using (5) is the optimum in terms of consumption rates. Moreover, if $E^{i *}$ is an optimum in terms of consumption rates, then the sequence $\left\{C^{i *}, K^{i *}\right\}$ constructed according to (6) is the optimum in terms of consumption levels. Thus, for each agent there is a one-to-one correspondence between the optimum in terms of consumption levels and the optimum in terms of consumption rates. It follows that there is a unique optimum in terms of consumption rates for each agent.

\footnotetext{
${ }^{10}$ We apply the change of the control variable similar to that of Phelps and Pollak (1968), and Peleg and Yaari (1973). They used as control variable savings rate, which in the Ramsey model is naturally related to consumption rate: $k_{t+1} / f\left(k_{t}\right)=s_{t}=1-e_{t}$. However, in the decision-making context it seems reasonable to use consumption rate instead of savings rate. Note also that in the apparent economic interpretation of the Ramsey model with common consumption, the common property resource problem, consumption rate $e_{t}$ becomes harvest or extraction rate.
} 
Intertemporal discounted utility in the Ramsey model is maximized "once-and-for-all" at the initial moment of time in an essentially atemporal way. However, choices in the real world can hardly be regarded as "once-and-for-all" choices among specific plans of actions. Instead, they are sequential step-by-step choices based on the presently available opportunities. To illustrate this point, Koopmans (1967) uses a metaphor of ascending a mountain covered with fog. In these circumstances, instead of looking for a "largely invisible optimal path", it is better to take small steps towards the top using only local information. This metaphor leads to an important recommendation: "it is desirable that models of optimal growth be designed so as to require, and make use of, only information actually or potentially available at the time of decisions affecting growth" (Koopmans, 1967).

The difference between the two types of decision-making can be described as the problem of precommitment. The "once-and-for-all" solution to the Ramsey model is optimal provided the individual can credibly commit to implement her decisions in the future. There arises a natural question, what is the optimal strategy for the decision-maker if she cannot precommit her future behavior?

To answer this question, Strotz (1956) (see also Pollak, 1968) considered timeinconsistent preferences and introduced the "strategy of consistent planning": the agent should choose the best plan she would actually follow. A consistent plan is the solution in the dynamic programming sense (see Bellman, 1957). Each period the individual chooses the optimal policy under the assumption that future policies are optimally chosen. It is shown that in the Ramsey model the consistent plan coincides with the "once-and-for-all" solution.

Phelps and Pollak (1968), also in the context of time-inconsistent preferences, propose another strategy that can be used if the decision-maker cannot precommit her future behavior. They note that it is convenient to study sequential step-by-step choices in the Ramsey model using savings rate as control variable. They introduce the sequence of savings rates which is the equilibrium in the Nash sense: savings rate in each period is optimally chosen provided all other savings rates are also optimal. Clearly, such an equilibrium has the following consistency property: in any period there is no motivation for the individual to change or to regret her action, given her actions in all other periods.

Note that the sequence of savings rates which is optimal in the dynamic programming sense, i.e., the "once-and-for-all" solution, is always the Nash equilibrium. However, the converse need not be true. The Nash equilibrium sequence of savings rates need not coincide with the "once-and-for-all" solution in terms of savings rates. This actually means that the Nash equilibrium may exist in more general circumstances. Indeed, Peleg and Yaari (1973) use savings rate as control variable in the optimal growth model in which utility changes over time. While in their framework the "once-and-for-all" solution does not, in general, exist, they prove that there exists the Nash equilibrium sequence of savings rates.

Unlike the scholars mentioned above, we are not interested in the time-inconsistency issues. We consider the Ramsey model with time-consistent preferences. Our goal is to study the correspondence between the two solutions. One of the results of Phelps and Pollak (1968) is that in the model with the CES utility function and the AK production function the "once-and-for-all" solution (in terms of savings rates) coincides with the Nash equilibrium sequence of savings rates. We show that this result is valid for more general forms of utility and production functions.

Consider problem (8) for an arbitrary agent, and omit the index $i$ for the simplicity of 
notation. Suppose that the optimal sequence of consumption rates is not chosen "onceand-for-all" at time 0. Instead, the decision-making process is as follows: at time $\tau$ the agent chooses consumption rate $e_{\tau}$. This decision is made under given available capital stock $k_{\tau}$ and some expectations about future consumption rates. The resulting value, which may depend on $k_{\tau}$ and expectations, we call a time $\tau$ temporary optimum. Clearly, the new capital stock $k_{\tau+1}$ is fully determined by $k_{\tau}$ and the time $\tau$ temporary optimum $e_{\tau}$. Then, at time $\tau+1$, the agent chooses $e_{\tau+1}$ under given $k_{\tau+1}$ and expectations about future consumption rates, and so on.

This step-by-step decision-making procedure seems realistic and is in line with Koopmans's recommendation. However, there arises a question of whether the result of this procedure is optimal for the agent. We define a step-by-step intertemporal optimum as the sequence, each element of which is a time $\tau$ temporary optimum given the current capital stock and provided the agent has perfect foresight about future consumption rates. We show that a step-by-step intertemporal optimum coincides with the optimum in terms of consumption rates. In other words, if the agent at each date, starting from $t=0$, sequentially makes the decisions under perfect foresight, then the consumption rate $e_{\tau}$ chosen at time $\tau$ coincides with the consumption rate $e_{\tau}$ chosen at time 0 "once-and-for-all".

Formally, let $\tau$ be an arbitrary point in time. Denote

$$
E_{\tau, t}=\left\{e_{\tau}, e_{\tau+1}, \ldots, e_{t}\right\}
$$

Suppose the capital stock is $k_{\tau}$, and the agent has some expectations about future consumption rates represented by a finite or infinite sequence $E_{\tau+1, T}=\left\{e_{t}\right\}_{t=\tau+1}^{T}$. We assume that agent's expectations are non-degenerate, i.e., $0<e_{t}<1$ for all $t$ (except the terminal time $T$ in the finite horizon problem). Then the objective function in the time $\tau$ optimization problem is

$$
V_{\tau}\left(k_{\tau}, e_{\tau}, E_{\tau+1, T}\right)=\sum_{t=\tau}^{T} \delta^{t-\tau} u\left(e_{t} f\left(\left(1-e_{t-1}\right) f\left(\left(1-e_{t-2}\right) f\left(\ldots f\left(k_{\tau}\right)\right)\right)\right)\right) .
$$

Definition. Given $k_{\tau}$ and non-degenerate expectations $E_{\tau+1, T}$, we call $e_{\tau}^{*}\left(k_{\tau}, E_{\tau+1, T}\right)$ a time $\tau$ temporary optimum if it is a solution to the one-dimensional optimization problem:

$$
\max _{0 \leq e_{\tau} \leq 1} V_{\tau}\left(k_{\tau}, e_{\tau}, E_{\tau+1, T}\right)
$$

If $T<+\infty$, a terminal time $T$ optimum $e_{T}^{*}$ is a solution to the problem

$$
\max _{0 \leq e_{T} \leq 1} V_{T}\left(k_{T}, e_{T}\right)=u\left(e_{T} f\left(k_{T}\right)\right),
$$

under given $k_{T}$. It is immediately clear that a terminal time $T$ optimum is the same for all agents, and $e_{T}^{*}=1$.

It follows from (6) that every state variable in the initial (time 0 ) problem, $k_{\tau+1}$ for $\tau \in \mathbb{T}$, is actually determined by the sequence of previously chosen consumption rates $E_{0, \tau}=\left\{e_{0}, e_{1}, \ldots, e_{\tau}\right\}$. For a given $k_{0}>0$, let us denote $k_{0,0}=k_{0}$, and recursively define the functions $k_{0, \tau}(\cdot, \cdot)$ as:

$$
\begin{gathered}
k_{0,1}\left(k_{0}, E_{0,0}\right)=\left(1-e_{0}\right) f\left(k_{0,0}\right) \\
\ldots \\
k_{0, \tau}\left(k_{0}, E_{0, \tau-1}\right)=\left(1-e_{\tau-1}\right) f\left(k_{0, \tau-1}\left(k_{0}, E_{0, \tau-2}\right)\right), \\
\ldots
\end{gathered}
$$


Definition. We call a sequence $E_{0, T}^{*}=\left\{e_{\tau}^{*}\right\}_{\tau=0}^{T}$ a step-by-step intertemporal optimum if

$$
e_{\tau}^{*}=e_{\tau}^{*}\left(k_{0, \tau}\left(k_{0}, E_{0, \tau-1}^{*}\right), E_{\tau+1, T}^{*}\right), \quad \tau \in \mathbb{T},
$$

and $0<e_{\tau}^{*}<1$ for all $\tau$ except the terminal time $T$ in the finite horizon problem.

That is, every element $e_{\tau}^{*}$ of a step-by-step intertemporal optimum is a time $\tau$ temporary optimum provided that all previous consumption rates were chosen according to the same procedure, $k_{\tau}=k_{0, \tau}\left(k_{0}, E_{0, \tau-1}^{*}\right),{ }^{11}$ and under perfect foresight about future consumption rates. Conditions $e_{\tau}^{*} \neq 0$ and $e_{\tau}^{*} \neq 1$ (for all $\tau$ except the terminal time $T$ in the finite horizon problem) rule our degenerate optima. Formally speaking, there can be step-by-step intertemporal optima of the form $\{1,1, \ldots\}$, which are clearly of no interest.

The construction of a step-by-step intertemporal optimum is an essentially dynamic process. At time 0 , given the initial capital stock $k_{0}$, the agent maximizes the objective function $V_{0}\left(k_{0}, e_{0}, E_{1, T}\right)$ with respect to $e_{0}$, under some expectations about future consumption rates, $E_{1, T}$. The solution of this problem, a time 0 temporary optimum, generically depends on these expectations. If the agent has perfect foresight at time 0 , and the solution is interior, then the outcome of this procedure, $e_{0}^{*}$, is the first element in a step-by-step intertemporal optimum.

Then, at time 1 , the available capital stock $k_{1}$ aggregates the decision made at time 0 . The agent knows the value of $k_{1}$, no matter whether the decision at time 0 was made under perfect foresight or not. Given $k_{1}$ the agent maximizes the objective function $V_{1}\left(k_{1}, e_{1}, E_{2, T}\right)$ with respect to $e_{1}$ under some expectations ${ }^{12}$ about future consumption rates, $E_{2, T}$. Thus the agent obtains a time 1 temporary optimum. If $k_{1}$ is determined by $e_{0}^{*}$, the agent has perfect foresight at time 1 , and the solution is interior, then the outcome $e_{1}^{*}$ is the second element in a step-by-step intertemporal optimum.

This process continues infinitely (if $T=\infty$ ), or until the terminal time $T$ (if $T<\infty$ ). At every date $\tau$ the agent considers the stock $k_{\tau}$ as given and solves the time $\tau$ optimization problem under some expectations about future consumption rates. The agent finds the consumption rate $e_{\tau}$, imposing no requirements on the past and future decisions, and treating them as given. If at each date, starting from time 0 , the agent acts sequentially under perfect foresight, and all the solutions are interior, then the resulting sequence is a step-by-step intertemporal optimum.

Note the important distinction between the described step-by-step procedure and dynamic programming. In the dynamic programming approach every time $\tau$ decision looks as if it is made separately from future decisions. However, Bellman's principle of optimality emphasizes optimality of future decisions and presupposes that the decision at time $\tau$ is made under explicit assumption that all future decisions are also made optimally. Thus the optimal solution today is essentially based on the optimal behaviour in the future. On the contrary, in the described above step-by-step optimization procedure future decisions are not supposed to be optimal. The emphasis is placed on perfect foresight.

It should be noticed that technically a step-by-step intertemporal optimum for agent $i$ coincides with the non-degenerate outcome of coordinate-wise maximization of the objective function (7). Indeed, the latter is a sequence $\left\{e_{\tau}^{*}\right\}_{\tau=0}^{\infty}$ such that each $e_{\tau}^{*}$ is a solution

\footnotetext{
${ }^{11}$ Note that the sequence $E_{0, \tau-1}^{*}$ contains the first $\tau$ elements from the step-by-step intertemporal optimum $E_{0, T}^{*}$, i.e., $E_{0, \tau-1}^{*}=\left\{e_{t}^{*}\right\}_{t=0}^{\tau-1}$.

${ }^{12}$ Expectations about consumption rates formed at time $\tau+1$ may not be the same as expectations formed at time $\tau$.
} 
to the atemporal problem

$$
\max _{0 \leq e_{\tau} \leq 1} \mathcal{V}^{i}\left(e_{0}^{*}, \ldots, e_{\tau-1}^{*}, e_{\tau}, e_{\tau+1}^{*}, \ldots\right)
$$

That is, $e_{\tau}^{*}$ is a result of maximization of $\mathcal{V}^{i}\left(e_{0}, e_{1}, \ldots\right)$ with respect to $e_{\tau}$, provided all other variables are already chosen according to the same procedure. ${ }^{13}$

Clearly, if a sequence is the optimum in terms of consumption rates (the "once-and-forall" solution), then this sequence is also obtained by coordinate-wise maximization. There arises a question, is the opposite also true, i.e., is the sequence obtained by coordinate-wise maximization an optimum in terms of consumption rates?

The following proposition shows that the answer to this question is positive in the finite horizon Ramsey model. The same result in the infinite horizon problem holds with the additional requirement that the marginal instantaneous utility tends to infinity at zero not too fast. We say that the instantaneous utility function $u(c)$ satisfies regularity condition if there exists $\gamma>0$ such that

$$
\lim _{c \rightarrow 0} c^{\gamma} u^{\prime}(c)=0
$$

Condition (11) means that the derivative $u^{\prime}(c)$ tends to infinity at $c \rightarrow 0$ no faster than any power function. ${ }^{14}$ For instance, functions $u(c)=\ln c$ and $u(c)=c^{\theta} / \theta$ for $\theta<1$, which are widely used in the literature, meet this regularity requirement. Moreover, every instantaneous utility function defined at zero (i.e., defined over $\mathbb{R}_{+}$) also satisfies (11).

Proposition 1. Consider the finite horizon Ramsey model or the infinite horizon Ramsey model with the instantaneous utility function that satisfies (11). A step-by-step intertemporal optimum coincides with the optimum in terms of consumption rates.

Proof. See Appendix A.

It follows that there exists a unique (non-degenerate) step-by-step intertemporal optimum for each agent. It coincides with the unique optimum in terms of consumption rates, and corresponds to the unique optimum in terms of consumption levels for this agent.

\section{$5 \quad$ Voting over consumption rates}

\subsection{Intertemporal majority voting: infinite horizon example}

Let us provide another simple example to gain some intuition about our voting approach. Consider the infinite horizon problem with the same logarithmic instantaneous utility function for all agents and the Cobb-Douglas production function. Given $k_{0}>0$, the optimization problem in terms of consumption levels for agent $i$ is as follows:

$$
\max \sum_{t=0}^{\infty} \delta_{i}^{t} \ln c_{t},
$$

s. t. $\quad c_{t}+k_{t+1}=k_{t}^{\alpha}, \quad c_{t} \geq 0, \quad k_{t+1} \geq 0, \quad t=0,1, \ldots$

\footnotetext{
${ }^{13}$ This is exactly the Nash equilibrium sequence of consumption rates.

${ }^{14}$ The class of instantaneous utility functions that satisfy this requirement is very similar to the class singled out and considered by Ekeland and Scheinkman (1986).
} 
The optimization problem in terms of consumption rates $e_{t}=\frac{c_{t}}{k_{t}^{\alpha}}$ for agent $i$ takes the form

$$
\max \left\{\ln \left(e_{0} k_{0}^{\alpha}\right)+\delta_{i} \ln \left(e_{1}\left(1-e_{0}\right)^{\alpha} k_{0}^{\alpha^{2}}\right)+\delta_{i}^{2} \ln \left(e_{2}\left(1-e_{1}\right)^{\alpha}\left(1-e_{0}\right)^{\alpha^{2}} k_{0}^{\alpha^{3}}\right)+\ldots\right\} .
$$

It is well-known that the savings rate of agent $i$ in this problem is constant and equal to $\alpha \delta_{i}$. Therefore her optimal consumption rate is also constant over time and equal to $1-\alpha \delta_{i}$. Hence the optimum in terms of consumption rates for agent $i$ is given by

$$
\left\{1-\alpha \delta_{i}, 1-\alpha \delta_{i}, \ldots\right\}
$$

Consider intertemporal majority voting over consumption rates. Let $\tau$ be an arbitrarily chosen point in time. Suppose the economy is at the state $k_{\tau}>0$, and agents have some expectations about future consumption rates, $\left\{e_{t}\right\}_{t=\tau+1}^{\infty}$.

The objective function of agent $i$ in voting over $e_{\tau}$ is given by

$$
\begin{gathered}
\ln \left(e_{\tau}\left(k_{\tau}\right)^{\alpha}\right)+\delta_{i} \ln \left(e_{\tau+1}\left(1-e_{\tau}\right)^{\alpha}\left(k_{\tau}\right)^{\alpha^{2}}\right) \\
+\delta_{i}^{2} \ln \left(e_{\tau+2}\left(1-e_{\tau+1}\right)^{\alpha}\left(1-e_{\tau}\right)^{\alpha^{2}}\left(k_{\tau}\right)^{\alpha^{3}}\right)+\ldots= \\
\ln e_{\tau}+\alpha \delta_{i} \ln \left(1-e_{\tau}\right)+\alpha^{2} \delta_{i}^{2} \ln \left(1-e_{\tau}\right)+\ldots+\Gamma_{\tau}^{i}= \\
\ln e_{\tau}+\alpha \delta_{i}\left(1+\alpha \delta_{i}+\ldots\right) \ln \left(1-e_{\tau}\right)+\Gamma_{\tau}^{i},
\end{gathered}
$$

where

$$
\Gamma_{\tau}^{i}=\ln \left(\left(k_{\tau}\right)^{\alpha}\right)+\delta_{i} \ln \left(e_{\tau+1}\left(k_{\tau}\right)^{\alpha^{2}}\right)+\delta_{i}^{2} \ln \left(e_{\tau+2}\left(1-e_{\tau+1}\right)^{\alpha}\left(k_{\tau}\right)^{\alpha^{3}}\right)+\ldots
$$

depends on $k_{\tau}$ and expectations, but not on the variable over which agents vote.

By maximizing this objective function with respect to $e_{\tau}$, agent $i$ obtains her preferred time $\tau$ consumption rate. Solving the equation

$$
\frac{d}{d e_{\tau}}\left(\ln e_{\tau}+\frac{\alpha \delta_{i}}{1-\alpha \delta_{i}} \ln \left(1-e_{\tau}\right)\right)=0
$$

we find that the preferred time $\tau$ consumption rate for agent $i$ is $1-\alpha \delta_{i}$. Note that in this example due to the log-linear utility functions and the Cobb-Douglas production function, the preferred time $\tau$ consumption rate for each agent is independent of expectations. ${ }^{15}$ Moreover, for each agent the preferred time $\tau$ consumption rate coincides with the optimal consumption rate.

It is clear that preferences of agents in one-dimensional voting over the time $\tau$ consumption rate are single-peaked and the preferred consumption rate negatively depends on $\delta_{i}$. By the median voter theorem, the winner in majority voting over the time $\tau$ consumption rate is the preferred consumption rate for the median agent, $1-\alpha \delta_{\text {med }}$.

Thus we obtain the sequence $\left\{1-\alpha \delta_{\text {med }}, 1-\alpha \delta_{\text {med }}, \ldots\right\}$, each element of which is a Condorcet winner in voting over the corresponding consumption rate. It is natural to call this sequence an intertemporal voting equilibrium. Clearly, an intertemporal voting equilibrium coincides with the optimum in terms of consumption rates for the agent with the median discount factor.

\footnotetext{
${ }^{15}$ In a number of papers (see Borissov et al., 2014a,b; Borissov and Pakhnin, 2014), this fact is used to generalize the considered example to voting in a dynamic general equilibrium framework. Because of the log-linear utility functions and the Cobb-Douglas production function, it is possible to find a closedform solution for the preferred policy of each agent, and explicitly calculate the winner in intertemporal majority voting.
} 


\subsection{Voting equilibria}

Let us describe formally intertemporal majority voting over consumption rates. We define our voting procedure in two stages. First, for an arbitrary point in time $\tau$ we define a time $\tau$ (temporary) voting equilibrium. This is a Condorcet winner in voting over the current consumption rate under given current state of the economy $k_{\tau}>0$ and expectations about future consumption rates. Secondly, we use the fact that the current state of the economy contains all relevant information about past decisions and define an intertemporal voting equilibrium. It is a sequence, each element of which is a time $\tau$ voting equilibrium provided agents have perfect foresight about future consumption rates.

Consider an arbitrary point in time $\tau$. Suppose the capital stock is $k_{\tau}>0$, and agents have some expectations about future consumption rates, $E_{\tau+1, T}=\left\{e_{t}\right\}_{t=\tau+1}^{T}{ }^{16} \mathrm{We}$ assume that expectations are non-degenerate $\left(0<e_{t}<1\right.$ for all $t$ except the terminal time $T$ in the finite horizon problem). The objective function of agent $i$ in voting over the time $\tau$ consumption rate is of the form (9):

$$
V_{\tau}^{i}\left(k_{\tau}, e_{\tau}, E_{\tau+1, T}\right)=\sum_{t=\tau}^{T} \delta_{i}^{t-\tau} u_{i}\left(e_{t} f\left(\left(1-e_{t-1}\right) f\left(\left(1-e_{t-2}\right) f\left(\ldots f\left(k_{\tau}\right)\right)\right)\right)\right) .
$$

In the context of voting behaviour, we call a time $\tau$ temporary optimum for agent $i$, i.e., a solution to the following one-dimensional optimization problem,

$$
\max _{0 \leq e_{\tau} \leq 1} V_{\tau}^{i}\left(k_{\tau}, e_{\tau}, E_{\tau+1, T}\right)
$$

the preferred time $\tau$ consumption rate for agent $i$. It follows that in the finite horizon problem the preferred time $T$ consumption rate is the same for all agents and equal to $e_{T}^{i}=1$.

Definition. We call $e_{\tau}^{v *}\left(k_{\tau}, E_{\tau+1, T}\right)$ a time $\tau$ voting equilibrium if it is a Condorcet winner in one-dimensional voting over the time $\tau$ consumption rate, where non-degenerate expectations $E_{\tau+1, T}$ are considered as given.

Clearly in the finite horizon case at time $T$ agents vote unanimously, and the terminal time $T$ voting equilibrium is $e_{T}^{v *}=1$.

We want to emphasize that our institutional framework is well-defined without any restrictions on agents' expectations. The expectations may not be the same for all agents, and may not even be correct. In any case, even without perfect foresight, voting over the current consumption rate is a well-defined one-dimensional decision problem, and the following Proposition holds.

Proposition 2. For any non-degenerate expectations $E_{\tau+1, T}$, preferences of each agent in voting over the time $\tau$ consumption rate are strictly concave and single-peaked. Therefore, the median voter theorem applies.

Proof. It follows from Lemma 3, which is proved in Appendix B.

Hence at each given point in time there exists the instantaneous Condorcet winner, which is by definition a time $\tau$ voting equilibrium.

\footnotetext{
${ }^{16}$ For simplicity of notation, we drop the superscript $i$ from expectations, though as a general rule each agent has its own expectations.
} 
Now suppose that agents vote step by step starting from time 0 . At time 0 agents have some expectations about future consumption rates, $E_{1, T}$, and the initial capital stock $k_{0}$ is given. The winner in voting over the time 0 consumption rate, time 0 voting equilibrium $e_{0}^{v *}\left(k_{0}, E_{1, T}\right)$, generically depends on expectations. Then, at time 1 , all relevant information about the decision made at time 0 is gathered in the new capital stock, since $k_{1}=\left(1-e_{0}^{v *}\left(k_{0}, E_{1, T}\right)\right) f\left(k_{0}\right)$. Agents vote over the time 1 consumption rate given $k_{1}$ and some expectations about future consumption rates, $E_{2, T}$. And so on. At every date $\tau$, the current capital stock $k_{\tau}$ is unambiguously determined by the past voting decisions. Hence for every $\tau \in \mathbb{T}, k_{\tau+1}$ is a function of $k_{0}$ and the past values of consumption rates $E_{0, \tau}$, given by (10).

Definition. We call a sequence $E_{0, T}^{v *}=\left\{e_{\tau}^{v *}\right\}_{\tau=0}^{T}$ an intertemporal voting equilibrium starting from $k_{0}>0$ if

$$
e_{\tau}^{v *}=e_{\tau}^{v *}\left(k_{0, \tau}\left(k_{0}, E_{0, \tau-1}^{v *}\right), E_{\tau+1, T}^{v *}\right), \quad \tau \in \mathbb{T},
$$

and $0<e_{\tau}^{v *}<1$ for all $\tau$ except the terminal time $T$ in the finite horizon problem.

Thus $E_{0, T}^{v *}=\left\{e_{\tau}^{v *}\right\}_{\tau=0}^{T}$ is an intertemporal voting equilibrium if each $e_{\tau}^{v *}$ is a time $\tau$ voting equilibrium under $k_{\tau}=k_{0, \tau}\left(k_{0}, E_{0, \tau-1}^{v *}\right){ }^{17}$ and perfect foresight about future consumption rates, $E_{\tau+1, T}^{v *}=\left\{e_{t}^{v *}\right\}_{t=\tau+1}^{T}$. Conditions $0<e_{\tau}^{v *}<1$ (for all $\tau$ except the terminal time $T$ in the finite horizon problem) rule our degenerate intertemporal voting equilibria of the form $\{1,1, \ldots\}$. We are interested only in the non-degenerate intertemporal voting equilibria.

Technically, an intertemporal voting equilibrium is a sequence, every element of which is chosen by a majority of agents provided all other consumption rates are already chosen according to the same procedure. Hence an intertemporal voting equilibrium is essentially a Kramer-Shepsle equilibrium.

\section{Intertemporal voting equilibrium: identical instanta- neous utility functions}

Consider the special, but important case where all agents have the same instantaneous utility function $u(c)$, so that agents are heterogeneous only in their time preferences. Let us show that the intertemporal voting equilibrium is the optimum in terms of consumption rates for the median agent (i.e., for the agent with the median discount factor).

Proposition 3. Suppose all agents have the same instantaneous utility function and the same expectations. A time $\tau$ voting equilibrium exists, is unique, and coincides with the preferred time $\tau$ consumption rate for the agent with the median discount factor.

Proof. Here we give only a sketch the proof. For the details, see Appendix B.

It follows from Lemma 3 that preferences of each agent in voting over the time $\tau$ consumption rate are strictly concave and therefore single-peaked. We also prove (see Lemma 4) that if agents have the same instantaneous utility function and the same expectations, then higher values of the discount factor correspond to lower values of the preferred time $\tau$ consumption rate. Hence by the median voter theorem, a time $\tau$ voting equilibrium is the preferred consumption rate for the agent with the median discount factor.

\footnotetext{
${ }^{17}$ It should be noticed that the sequence $E_{0, \tau-1}^{v *}$ contains the first $\tau$ elements from the intertemporal voting equilibrium $E_{0, T}^{v *}$. That is, $E_{0, \tau-1}^{v *}=\left\{e_{t}^{v *}\right\}_{t=0}^{\tau-1}$.
} 
Combining Propositions 1 and 3, we can formulate the following Theorem.

Theorem 1. Suppose all agents have the same instantaneous utility function. Consider the finite horizon Ramsey model or the infinite horizon Ramsey model with the instantaneous utility function that satisfies (11). An intertemporal voting equilibrium starting from any $k_{0}>0$ is unique and coincides with the optimum in terms of consumption rates for the agent with the median discount factor.

Proof. Proposition 3 states that a time $\tau$ voting equilibrium is the time $\tau$ temporary optimum for the median agent. Taking into account the definition of a step-by-step intertemporal optimum, it follows from Proposition 1 that an intertemporal voting equilibrium is the optimum in terms of consumption rates for the agent with the median discount factor.

By (6), there is a one-to-one correspondence between the optimum in terms of consumption rates and the optimum in terms of consumption levels. It follows that there is a one-to-one correspondence between the intertemporal voting equilibrium and the optimum in terms of consumption levels for the median agent. Thus the proposed voting mechanism yields the optimum in terms of consumption levels for the median agent as the stable outcome.

\section{Balanced-growth and steady-state voting equilibria}

In this section we study an intertemporal voting equilibrium in the general case. Suppose that agents differ both in their time preferences and utility functions. Clearly, there is no reason to expect that the result of Theorem 1 still holds in this case. There are no results for the general case even when all agents have CES utility functions. Because of multidimensional heterogeneity, it is in principle impossible to claim that an intertemporal voting equilibrium is determined by the discount factor alone.

However, there are two special cases in which we are able to provide some results even with multi-dimensional heterogeneity. The first is the case of a linear production function and CES utility functions. Under these assumptions, we define a balanced-growth voting equilibrium. The second is the case of a strictly concave production function where we define and characterize a steady-state voting equilibrium. It is clear that these two cases are essentially different.

\subsection{Linear production function}

Consider the CES utility function:

$$
u_{i}(c)=\left\{\begin{array}{l}
\frac{c^{1-\rho_{i}}}{1-\rho_{i}}, \quad \text { if } 0<\rho_{i}<+\infty, \quad \rho_{i} \neq 1, \\
\ln c, \quad \text { if } \rho_{i}=1,
\end{array}\right.
$$

and the AK production function,

$$
f(k)=A k, \quad A>0 .
$$

The optimization problem in terms of consumption levels for agent $i$ is as follows:

$$
\begin{gathered}
\max \sum_{t=0}^{\infty} \delta_{i}^{t} u_{i}\left(c_{t}\right), \\
\text { s. t. } \quad c_{t}+k_{t+1}=A k_{t}, \quad c_{t} \geq 0, \quad k_{t+1} \geq 0, \quad t=0,1, \ldots,
\end{gathered}
$$


where $k_{0}$ is given.

We assume that for any $i$,

$$
\delta_{i} A^{1-\rho_{i}}<1
$$

The first-order conditions for the optimal solution $\left\{c_{t}^{i *}, k_{t+1}^{i *}\right\}_{t=0}^{\infty}$ can be expressed as

$$
\frac{u_{i}^{\prime}\left(c_{t}^{i *}\right)}{u_{i}^{\prime}\left(c_{t+1}^{i *}\right)}=\left(\frac{c_{t+1}^{i *}}{c_{t}^{i *}}\right)^{\rho_{i}}=\delta_{i} f^{\prime}\left(k_{t+1}^{i *}\right)=\delta_{i} A
$$

and hence

$$
\begin{array}{ll}
c_{t+1}^{i *}=\left(\delta_{i} A\right)^{\frac{1}{\rho_{i}}} c_{t}^{i *}, & t=0,1, \ldots, \\
k_{t+1}^{i *}=\left(\delta_{i} A\right)^{\frac{1}{\rho_{i}}} k_{t}^{i *}, & t=0,1, \ldots
\end{array}
$$

Therefore, regardless of initial conditions, optimal consumption and the capital stock for agent $i$ grow at a constant rate $\gamma_{i}$ given by

$$
1+\gamma_{i}=\left(\delta_{i} A\right)^{\frac{1}{\rho_{i}}} .
$$

To ensure that the discounted utility is finite, the condition $1+\gamma_{i}<A$ should hold, which is equivalent to (14). Thus for each agent $i$ and for any $k_{0}$ there is a unique optimal path which is a balanced-growth optimum.

Consider the constant sequence of consumption rates, $E=\{e, e, \ldots\}$. Then for any initial state $k_{0}$, consumption and the capital stock from the corresponding sequence $\{C, K\}$ grow at a constant rate $\gamma=(1-e) A-1$ :

$$
\begin{gathered}
k_{t+1}=(1-e) A k_{t}, \quad t=0,1, \ldots, \\
c_{t+1}=e A k_{t+1}=e(1-e) A^{2} k_{t}=(1-e) A c_{t}, \quad t=0,1, \ldots
\end{gathered}
$$

Now let us introduce the notion of a balanced-growth voting equilibrium.

Definition. We call $e^{*}$ a balanced-growth voting equilibrium starting from $k_{0}>0$ if the sequence $\left\{e^{*}, e^{*}, \ldots\right\}$ is an intertemporal voting equilibrium starting from $k_{0}$.

Clearly, agents' heterogeneity is multi-dimensional, since agents differ both in the discount factors and in the elasticities of intertemporal substitution. However, due to the linear production function, agents' heterogeneity can in some sense be considered as one-dimensional, because agents are naturally characterized by their growth rates that combine both heterogeneity parameters. The following theorem shows that the outcome of voting is fully determined by the preferences of the agent with the median growth rate.

Theorem 2. For any $k_{0}$, there is a unique balanced-growth voting equilibrium. It is given by

$$
e^{*}=1-\frac{1}{A}\left(1+\gamma_{m e d}\right),
$$

where $\gamma_{\text {med }}$ is the median growth rate.

Proof. See Appendix C.

Note that $e^{*}$ is the optimal consumption rate for the agent with the median growth rate $\gamma_{\text {med }}$. Moreover, it is easily seen that the sequence $\left\{C^{*}, K^{*}\right\}$ corresponding to the sequence $E^{*}=\left\{e^{*}, e^{*}, \ldots\right\}$ is the balanced-growth optimum for this agent. 


\subsection{Strictly concave production function}

Now let us define a steady-state voting equilibrium in the model with the production function that satisfies (3).

If the sequence of consumption rates is constant, $E=\{e, e, \ldots\}$, then the considered model becomes the Solow model with the unique non-degenerate steady-state capital stock $k(e)$ which is the positive solution to the following equation ${ }^{18}$ in $k$ :

$$
k=(1-e) f(k) .
$$

Definition. We call $e^{*}$ a steady-state voting equilibrium if the sequence $\left\{e^{*}, e^{*}, \ldots\right\}$ is an intertemporal voting equilibrium starting from $k_{0}=k\left(e^{*}\right)>0$.

Suppose first that all agents have the same instantaneous utility function that satisfies (11). It follows from Theorem 1 that for any $k_{0}$ there is a unique intertemporal voting equilibrium, which corresponds to the optimum for the agent with the median discount factor. Take as the initial capital stock the value $k^{*}$ determined by the "modified golden rule" for the median agent:

$$
\delta_{\text {med }} f^{\prime}\left(k^{*}\right)=1 .
$$

The optimum for the agent with the median discount factor starting from $k_{0}=k^{*}$ is her steady-state optimum. The corresponding optimum in terms of consumption rates is a constant sequence $E^{*}=\left\{e^{*}, e^{*}, \ldots\right\}$, and by Theorem 1 is a unique intertemporal voting equilibrium. Clearly, $e^{*}$ and $k^{*}$ are linked by equation (16). Hence $e^{*}$, the optimal consumption rate for the agent with the median discount factor $\delta_{m e d}$, is the unique steadystate voting equilibrium.

Since $k^{*}$ is given by the "modified golden rule" (17), and $e^{*}$ depends only on the median discount factor, the steady-state voting equilibrium does not depend on the instantaneous utility function of agents. This observation leads to the following theorem which holds in the general case where agents have different utility functions.

Theorem 3. There is a unique steady-state voting equilibrium. It is given by

$$
e^{*}=1-\frac{k^{*}}{f\left(k^{*}\right)},
$$

where $k^{*}$ is a solution to equation (17).

Proof. See Appendix D.

Note that $e^{*}$ is the optimal consumption rate for the agent with the median discount factor $\delta_{\text {med }}$. Clearly, the sequence $\left\{C^{*}, K^{*}\right\}$ corresponding to the sequence $E^{*}=$ $\left\{e^{*}, e^{*}, \ldots\right\}$ is the steady-state optimum for this agent.

It is well-known that in the Ramsey model with the production function that satisfies (3), the optimal path of each agent converges to the steady-state optimum. The steadystate consumption is fully determined by the production function and the steady-state capital stock. The steady-state capital stock, defined by the "modified golden rule", depends on the discount factor of the agent, and is independent of her instantaneous utility function.

Hence the optimum in terms of consumption rates for each agent converges to the steady-state optimum, which is determined by the discount factor of the agent and is

\footnotetext{
${ }^{18}$ The nonzero solution to the following equation exists if $(1-e) f^{\prime}(0)>1$.
} 
independent of her instantaneous utility function. Does in our framework every intertemporal voting equilibrium converge to the steady-state voting equilibrium? Is the outcome of voting in the general case eventually determined by the discount factors of the agents (more precisely, by the median discount factor)? It is a topic for further research, whether it is possible to generalize the standard Ramsey model results to voting equilibria.

\section{Conclusion}

The problem of collective choice naturally arises in many economic applications. It is reasonable to conjecture that the outcome of majority voting coincides with the optimum for the "median" agent whenever this notion is well-defined. However, if the choice space is multi-dimensional, there are difficulties in achieving this outcome.

In this paper we study a Ramsey-type model with common consumption and agents who have different discount factors and utility functions. Agents choose consumption stream from the set of all feasible consumption streams by majority voting. It is known that there is no Condorcet winner in voting over feasible consumption streams in the Ramsey model. We propose a simple and natural voting rule which picks the optimum for the median agent as a stable outcome in many important cases.

Our intertemporal majority voting is based on two principles. First, voting over the multi-dimensional sequence is replaced by the step-by-step sequence of the onedimensional votes. Second, agents vote over the consumption rate instead of consumption level. We define a temporary voting equilibrium, which is a Condorcet winner in voting over the current consumption rate under some expectations about future consumption rates. Then we define an intertemporal voting equilibrium as a sequence, each element of which is a temporary voting equilibrium provided agents have perfect foresight about future consumption rates. From the technical point of view, an intertemporal voting equilibrium is a Kramer-Shepsle equilibrium in terms of consumption rates.

Our main result concerns the case where agents have the same instantaneous utility function and differ only in their discount factors. We prove that an intertemporal voting equilibrium exists, is unique, and coincides with the optimum in terms of consumption rates for the agent with the median discount factor. It follows that there is a one-to-one correspondence between the intertemporal voting equilibrium and the optimum in terms of consumption levels for the median agent. We thus show that even in the absence of a Condorcet winner there is a stable outcome of majority voting. Since this outcome is determined by the preferences of the agent with the median discount factor, it is both time-consistent and Pareto efficient.

We also consider the general framework in which agents differ in their utility functions, and analyze two special cases. In the case of a linear production function and CES utility functions, we define a balanced-growth voting equilibrium, prove its uniqueness and show that it is determined by the median growth rate. In the case of a strictly concave production function, we define a steady-state voting equilibrium, and show that it is unique and only depends on the median discount factor.

Our analysis suggests that in the case of a strictly concave production function the analogy with the standard Ramsey model may fruitfully be applied. One may conjecture that every intertemporal voting equilibrium converges to the steady-state voting equilibrium, and thus that the winner of the voting procedure eventually depends only on the discount factor. However, further research is needed to confirm or to reject this conjecture. 


\section{A Proof of Proposition 1}

\section{A.1 Useful notation}

Recall that

$$
E_{\tau, t}=\left\{e_{\tau}, e_{\tau+1}, \ldots, e_{t}\right\}
$$

Let for arbitrary $\tau$ and $t>\tau$,

$$
\begin{gathered}
k_{\tau, \tau}=k_{\tau}, \\
k_{\tau, \tau+1}\left(k_{\tau}, E_{\tau, \tau}\right)=\left(1-e_{\tau}\right) f\left(k_{\tau, \tau}\right), \\
\ldots, \\
k_{\tau, t+1}\left(k_{\tau}, E_{\tau, t}\right)=\left(1-e_{t}\right) f\left(k_{\tau, t}\left(k_{\tau}, E_{\tau, t-1}\right)\right) .
\end{gathered}
$$

Let also

$$
\begin{gathered}
f_{\tau, \tau}=f\left(k_{\tau}\right), \\
f_{\tau, \tau+1}\left(k_{\tau}, E_{\tau, \tau}\right)=f\left(\left(1-e_{\tau}\right) f_{\tau, \tau}\right), \\
\cdots \\
f_{\tau, t+1}\left(k_{\tau}, E_{\tau, t}\right)=f\left(\left(1-e_{t}\right) f_{\tau, t}\left(k_{\tau}, E_{\tau, t-1}\right)\right) .
\end{gathered}
$$

Thus for $t>\tau, k_{\tau, t}$ are linked with $f_{\tau, t}$ as follows:

$$
\begin{gathered}
k_{\tau, t+1}\left(k_{\tau}, E_{\tau, t}\right)=\left(1-e_{t}\right) f_{\tau, t}\left(k_{\tau}, E_{\tau, t-1}\right), \\
f_{\tau, t+1}\left(k_{\tau}, E_{\tau, t}\right)=f\left(k_{\tau, t+1}\left(k_{\tau}, E_{\tau, t}\right)\right) .
\end{gathered}
$$

For simplicity of notation, we often drop the arguments of these functions when they play no significant role. However, the reader should bear in mind that $f_{\tau, t+1}$ is a function of the $t+1$ consumption rates $\left\{e_{\tau}, e_{\tau+1}, \ldots, e_{t}\right\}$.

The derivatives of $f_{\tau, t+1}$ can be obtained using a chain rule of differentiation:

$$
\begin{gathered}
\frac{\partial f_{\tau, t+1}}{\partial e_{t}}=-f^{\prime}\left(k_{\tau, t+1}\right) f_{\tau, t}, \\
\frac{\partial f_{\tau, t+1}}{\partial e_{t-1}}=-f^{\prime}\left(k_{\tau, t+1}\right)\left(1-e_{t}\right) f^{\prime}\left(k_{\tau, t}\right) f_{\tau, t-1},
\end{gathered}
$$

It is clear that the derivative of $f_{\tau, t+1}\left(k_{\tau}, E_{\tau, t}\right)$ with respect to each consumption rate $\left\{e_{\tau}, e_{\tau+1}, \ldots, e_{t}\right\}$ is negative.

\section{A.2 The first-order conditions}

The solution to problem (4) for an arbitrary agent is characterized by the first-order conditions and the transversality condition. The first-order conditions are given by

$$
\frac{u^{\prime}\left(c_{t}\right)}{u^{\prime}\left(c_{t+1}\right)}=\delta f^{\prime}\left(k_{t+1}\right), \quad t=0,1, \ldots(T-1) \cdot{ }^{19}
$$

${ }^{19}$ The notation $t=0,1, \ldots(T-1)$ means that these equations hold for all $t$ except the terminal time $T$ in the finite horizon problem. 
The transversality condition in the finite horizon problem is as follows:

$$
k_{T+1}=0 .
$$

In the infinite horizon problem, the transversality condition is given by

$$
\lim _{t \rightarrow \infty} \delta^{t} u^{\prime}\left(c_{t}\right) k_{t+1}=0 .
$$

Let us show that the sequence $\left\{C^{*}, K^{*}\right\}$ which is constructed according to (6) and corresponds to a step-by-step intertemporal optimum $E^{*}$, satisfies the first-order conditions (19).

From the definition of a step-by-step intertemporal optimum we immediately obtain that a step-by-step intertemporal optimum $E^{*}$ satisfies the following system of equations:

$$
\frac{\partial V_{t}\left(k_{0, t}\left(k_{0}, E_{0, t-1}\right), e_{t}, E_{t+1, T}\right)}{\partial e_{t}}=0, \quad t=0,1, \ldots,(T-1) .
$$

Consider two adjacent equations of the system of equations (22), for $t=\tau$ and for $t=\tau+1$. The equation for $t=\tau$ is as follows:

$$
\frac{\partial u\left(e_{\tau} f_{0, \tau}\left(k_{0}, E_{0, \tau-1}\right)\right)}{\partial e_{\tau}}+\delta \frac{\partial u\left(e_{\tau+1} f_{0, \tau+1}\left(k_{0}, E_{0, \tau}\right)\right)}{\partial e_{\tau}}+\ldots=0
$$

Writing out derivatives, we obtain:

$$
\begin{gathered}
u^{\prime}\left(e_{\tau} f_{0, \tau}\right) f_{0, \tau}= \\
\delta u^{\prime}\left(e_{\tau+1} f_{0, \tau+1}\right) e_{\tau+1} f^{\prime}\left(k_{0, \tau+1}\right) f_{0, \tau}+ \\
\delta^{2} u^{\prime}\left(e_{\tau+2} f_{0, \tau+2}\right) e_{\tau+2} f^{\prime}\left(k_{0, \tau+2}\right)\left(1-e_{\tau+1}\right) f^{\prime}\left(k_{0, \tau+1}\right) f_{0, \tau}+ \\
\delta^{3} u^{\prime}\left(e_{\tau+3} f_{0, \tau+3}\right) e_{\tau+3} f^{\prime}\left(k_{0, \tau+3}\right)\left(1-e_{\tau+2}\right) f^{\prime}\left(k_{0, \tau+2}\right)\left(1-e_{\tau+1}\right) f^{\prime}\left(k_{0, \tau+1}\right) f_{0, \tau}+\ldots
\end{gathered}
$$

Dividing both parts of the above equation by $f_{0, \tau}>0$, we get

$$
\begin{gathered}
u^{\prime}\left(e_{\tau} f_{0, \tau}\right)= \\
\delta f^{\prime}\left(k_{0, \tau+1}\right)\left[e_{\tau+1} u^{\prime}\left(e_{\tau+1} f_{0, \tau+1}\right)+\left(1-e_{\tau+1}\right) \delta e_{\tau+2} u^{\prime}\left(e_{\tau+2} f_{0, \tau+2}\right) f^{\prime}\left(k_{0, \tau+2}\right)+\right. \\
\left.\left(1-e_{\tau+1}\right) \delta^{2}\left(1-e_{\tau+2}\right) e_{\tau+3} u^{\prime}\left(e_{\tau+3} f_{0, \tau+3}\right) f^{\prime}\left(k_{0, \tau+3}\right) f^{\prime}\left(k_{0, \tau+2}\right)+\ldots\right] .
\end{gathered}
$$

The equation for $t=\tau+1$ is as follows:

$$
\frac{\partial u\left(e_{\tau+1} f_{0, \tau+1}\left(k_{0}, E_{0, \tau}\right)\right)}{\partial e_{\tau+1}}+\delta \frac{\partial u\left(e_{\tau+2} f_{0, \tau+2}\left(k_{0}, E_{0, \tau+1}\right)\right)}{\partial e_{\tau+1}}+\ldots=0,
$$

or, equivalently (dividing both parts of the resulting equation by $f_{0, \tau+1}>0$ ),

$$
\begin{gathered}
u^{\prime}\left(e_{\tau+1} f_{0, \tau+1}\right)= \\
\delta e_{\tau+2} u^{\prime}\left(e_{\tau+2} f_{0, \tau+2}\right) f^{\prime}\left(k_{0, \tau+2}\right)+ \\
\delta^{2}\left(1-e_{\tau+2}\right) e_{\tau+3} u^{\prime}\left(e_{\tau+3} f_{0, \tau+3}\right) f^{\prime}\left(k_{0, \tau+3}\right) f^{\prime}\left(k_{0, \tau+2}\right)+\ldots
\end{gathered}
$$

The right-hand side of the above equation appears also in the right-hand side of equation (23). Substituting, we get

$$
\begin{gathered}
u^{\prime}\left(e_{\tau} f_{0, \tau}\right)= \\
\delta f^{\prime}\left(k_{0, \tau+1}\right)\left(e_{\tau+1} u^{\prime}\left(e_{\tau+1} f_{0, \tau+1}\right)+\left(1-e_{\tau+1}\right) u^{\prime}\left(e_{\tau+1} f_{0, \tau+1}\right)\right)= \\
\delta f^{\prime}\left(k_{0, \tau+1}\right) u^{\prime}\left(e_{\tau+1} f_{0, \tau+1}\right) .
\end{gathered}
$$


Applying this argument for $\tau=0,1, \ldots,(T-1)$, we obtain that system of equations (22) is equivalent to the system

$$
u^{\prime}\left(e_{t} f_{0, t}\right)=\delta f^{\prime}\left(k_{0, t+1}\right) u^{\prime}\left(e_{t+1} f_{0, t+1}\right), \quad t=0,1, \ldots,(T-1) .
$$

Now it is straightforward to see that the mapping defined by (6) converts the system of equations (24) to the system of the first-order conditions (19).

\section{A.3 The transversality condition}

It remains to show that the sequence $\left\{C^{*}, K^{*}\right\}$ corresponding to a step-by-step intertemporal optimum satisfies the transversality condition.

Consider the case $T<\infty$. Then the transversality condition (20) follows from the fact that the optimal extraction rate at the terminal time $T$ for every agent is $e_{T}^{i *}=1$. At the terminal time it is optimal to consume everything.

Consider the case $T=\infty$. Using (24), we can replace expressions of the form

$$
\delta^{t-(\tau+1)} u^{\prime}\left(e_{t} f_{0, t}\right) f^{\prime}\left(k_{0, t}\right) f^{\prime}\left(k_{0, t-1}\right) \cdot \ldots \cdot f^{\prime}\left(k_{0, \tau+1}\right)
$$

in the right-hand side of equation (23) with $u^{\prime}\left(e_{\tau} f_{0, \tau}\right)$. Hence equation (23) can be rewritten as follows:

$$
\begin{gathered}
u^{\prime}\left(e_{\tau} f_{0, \tau}\right)-e_{\tau+1} u^{\prime}\left(e_{\tau} f_{0, \tau}\right)- \\
\left(1-e_{\tau+1}\right) e_{\tau+2} u^{\prime}\left(e_{\tau} f_{0, \tau}\right)-\left(1-e_{\tau+1}\right)\left(1-e_{\tau+2}\right) e_{\tau+3} u^{\prime}\left(e_{\tau} f_{0, \tau}\right)- \\
\left(1-e_{\tau+1}\right)\left(1-e_{\tau+2}\right)\left(1-e_{\tau+3}\right) e_{\tau+4} u^{\prime}\left(e_{\tau} f_{0, \tau}\right)-\ldots= \\
u^{\prime}\left(e_{\tau} f_{0, \tau}\right)\left(1-e_{\tau+1}\right)\left(1-e_{\tau+2}\right)\left(1-e_{\tau+3}-\left(1-e_{\tau+3}\right) e_{\tau+4} u^{\prime}\left(e_{\tau} f_{0, \tau}\right)-\ldots\right)=0 .
\end{gathered}
$$

Thus we come to the condition

$$
u^{\prime}\left(e_{\tau} f_{0, \tau}\right)\left(1-e_{\tau+1}\right)\left(1-e_{\tau+2}\right)\left(1-e_{\tau+3}\right) \cdots=0 .
$$

Since $\tau$ is chosen arbitrarily, and $u^{\prime}>0$, it follows that

$$
\prod_{t=1}^{\infty}\left(1-e_{t}\right)=0
$$

which is equivalent to

$$
\sum_{t=1}^{\infty} e_{t}=+\infty .
$$

Now let us show that the sequence $\left\{c_{t}, k_{t+1}\right\}_{t=0}^{\infty}$ which is constructed according to (6) and corresponds to the solution to the system (22), always satisfies the transversality condition (21). Recall that

$$
c_{t}=e_{t} f\left(k_{t}\right) .
$$

Consider separately the model with the AK production function, and the model with the production function satisfying properties (3). 


\section{A.3.1 Linear production function}

Suppose $f(k)=A k, A \neq 0$. The first-order conditions state that

$$
A^{t} \delta^{t} u^{\prime}\left(c_{t}\right)=u^{\prime}\left(c_{0}\right) \quad t=1,2, \ldots
$$

Assume that the transversality condition (21) fails. Then there exist $T$ and $N>0$ such that for all $t>T, \delta^{t} u^{\prime}\left(c_{t}\right) k_{t+1} \geq N$. Using (27), we infer that for all $t>T$,

$$
\frac{k_{t+1}}{A^{t}} \geq \frac{N}{u^{\prime}\left(c_{0}\right)}
$$

It follows from (26) and (28) that for all $t>T$,

$$
e_{t+1}=\frac{c_{t+1}}{A k_{t+1}} \leq \frac{u^{\prime}\left(c_{0}\right)}{N} \frac{c_{t+1}}{A^{t+1}}
$$

Therefore, by (25),

$$
\sum_{t=T}^{\infty} \frac{c_{t+1}}{A^{t+1}}=+\infty .
$$

However, iterating the equation $c_{t}+k_{t+1}=A k_{t}$, we easily get

$$
c_{0}+\frac{c_{1}}{A}+\frac{c_{2}}{A^{2}}+\ldots \leq A k_{0} .
$$

Hence we arrive at a contradiction. The transversality condition (21) holds for $f(k)=A k$.

\section{A.3.2 Strictly concave production function}

Suppose that $f(k)$ satisfies the following properties:

$$
f(0)=0, \quad f^{\prime}(k)>0, \quad f^{\prime \prime}(k)<0, \quad \exists \bar{k}: f(\bar{k})=\bar{k}, \quad \delta_{\min } f^{\prime}(0)>1 .
$$

Our goal is to consider the possible dynamics of the sequence $\left\{c_{t}, k_{t+1}\right\}_{t=0}^{\infty}$. We begin with two important lemmas.

Lemma 1. Suppose that $\delta f^{\prime}\left(k_{T+1}\right)>1$ for some $T$, and $k_{T+1} \leq k_{T}$. Then the transversality condition (21) holds.

Proof. Let us show that the conditions of lemma imply $k_{t+1}<k_{t}$ for all $t \geq T$. Indeed, from $\delta f^{\prime}\left(k_{T+1}\right)>1$ and the first-order conditions we get $u^{\prime}\left(c_{T}\right)>u^{\prime}\left(c_{T+1}\right)$, or $c_{T+1}>c_{T}$. Moreover,

$$
k_{T+2}-k_{T+1}=\left(f\left(k_{T+1}\right)-f\left(k_{T}\right)\right)+\left(c_{T}-c_{T+1}\right)<0 .
$$

Thus $k_{T+2}<k_{T+1}$ and hence $\delta f^{\prime}\left(k_{T+2}\right)>1$. Repeating the argument, we infer that for all $t>T$,

$$
k_{t+1}<k_{T}, \quad \text { and } \quad u^{\prime}\left(c_{t}\right)<u^{\prime}\left(c_{T}\right) .
$$

Therefore, starting from $t=T$,

$$
\delta^{t} u^{\prime}\left(c_{t}\right) k_{t+1}<\delta^{t} u^{\prime}\left(c_{T}\right) k_{T}
$$

and

$$
\lim _{t \rightarrow \infty} \delta^{t} u^{\prime}\left(c_{t}\right) k_{t+1}=0 .
$$

This proves Lemma 1. 
Lemma 2. Suppose that there exists $T$ such that $\delta f^{\prime}\left(k_{t+1}\right) \leq 1$ for all $t \geq T$. Then the transversality condition (21) holds.

Proof. Since $\delta f^{\prime}(0)>1$, it follows that for $t>T, k_{t} \geq\left(f^{\prime}\right)^{-1}(1 / \delta)>0$. Hence $f\left(k_{t}\right) \geq$ $f\left(\left(f^{\prime}\right)^{-1}(1 / \delta)\right)>0$, and from $(26)$ we get

$$
c_{t}=e_{t} f\left(k_{t}\right) \geq e_{t} f\left(\left(f^{\prime}\right)^{-1}(1 / \delta)\right), \quad t>T .
$$

Therefore due to (25),

$$
\sum_{t=T}^{\infty} c_{t}=+\infty
$$

We claim that (29) is impossible if the transversality condition (21) fails. Indeed, since there is $\bar{k}$ such that $0<f(\bar{k})=\bar{k}<+\infty, k_{t}$ is bounded from above. Thus the failure of the transversality condition means that there exists $N>0$ such that for all $t>T$,

$$
\delta^{t} u^{\prime}\left(c_{t}\right) \geq N
$$

Let us show that from the regularity condition (11) and (30) it follows that $\sum_{t=T}^{\infty} c_{t}<+\infty$. We have:

$$
\delta^{t} u^{\prime}\left(c_{t}\right) \geq N \Leftrightarrow c_{t} \leq\left(u^{\prime}\right)^{-1}\left(N \delta^{-t}\right) \Leftrightarrow \sum_{t=T}^{\infty} c_{t} \leq \sum_{t=T}^{\infty}\left(u^{\prime}\right)^{-1}\left(N \delta^{-t}\right) .
$$

The series in the right-hand side of the last inequality converges if and only if the following integral converges at infinity:

$$
\int_{T}^{\infty}\left(u^{\prime}\right)^{-1}\left(N \delta^{-t}\right) d t=\int_{N \delta^{-T}}^{\infty} \frac{\left(u^{\prime}\right)^{-1}(y)}{y} d y .
$$

By the limit comparison test for improper integrals we infer that its convergence is equivalent to the existence of the limit

$$
\lim _{y \rightarrow \infty}\left(u^{\prime}\right)^{-1}(y) \cdot y^{\kappa}=L,
$$

where $0<L<\infty$, and $\kappa>0$. It follows from (11) that this limit exists, so we arrive at a contradiction with $(29)$.

This proves Lemma 2.

Now let us consider different cases that may arise. If $\delta f^{\prime}\left(k_{t+1}\right) \leq 1$ for all sufficiently large $t$, then the transversality condition (21) holds by Lemma 2 .

Suppose there exists $T$ such that $\delta f^{\prime}\left(k_{T+1}\right)>1$. Then there are only two possibilities. Either there exists $T_{1}>T$ such that $\delta f^{\prime}\left(k_{T_{1}+1}\right) \leq 1$ or $\delta f^{\prime}\left(k_{t+1}\right)>1$ for all $t>T$. In the former case, either $\delta f^{\prime}\left(k_{t+1}\right) \leq 1$ for all $t>T_{1}$ so that Lemma 2 holds or there exists $T_{2}>T_{1}$ such that $\delta f^{\prime}\left(k_{T_{2}+1}\right)>1$ in which case we are in the conditions of Lemma 1 . In the latter case, for all $t>T, u^{\prime}\left(c_{t}\right)<u^{\prime}\left(c_{T}\right)$ and $k_{t+1} \leq\left(f^{\prime}\right)^{-1}(1 / \delta)$. Therefore,

$$
\delta^{t} u^{\prime}\left(c_{t}\right) k_{t+1}<\delta^{t} u^{\prime}\left(c_{T}\right)\left(f^{\prime}\right)^{-1}(1 / \delta),
$$

which implies

$$
\lim _{t \rightarrow \infty} \delta^{t} u^{\prime}\left(c_{t}\right) k_{t+1}=0 .
$$

Thus we have described the possible dynamics of the sequence $\left\{c_{t}, k_{t+1}\right\}_{t=0}^{\infty}$ corresponding to the solution to the system of equations (22). We have shown that the transversality condition (21) is always satisfied. 


\section{B Proof of Propositions 2 and 3}

Consider how agents vote over the time $\tau$ consumption rate.

Lemma 3. For any given non-degenerate expectations $E_{\tau+1, T}$ preferences of each agent in voting over the time $\tau$ consumption rate are strictly concave and therefore single-peaked. Moreover, the preferred time $\tau$ consumption rate for agent $i$ is a solution to the equation

$$
\frac{\partial V_{\tau}^{i}\left(k_{\tau}, e_{\tau}, E_{\tau+1, T}\right)}{\partial e_{\tau}}=0, \quad \tau=0,1, \ldots(T-1),
$$

where the objective function $V_{\tau}^{i}\left(k_{\tau}, e_{\tau}, E_{\tau+1, T}\right)$ is given by (12).

Proof. Consider agent $i$ with the discount factor $\delta_{i}$ and the instantaneous utility function $u_{i}(c)$. Let us show that for all $\tau$ (except $\tau=T$ in the finite horizon case) and for any given non-degenerate expectations $E_{\tau+1, T}$, her preferred time $\tau$ consumption rate is a unique solution to equation (31).

Equation (31) can be rewritten as

$$
\begin{gathered}
u_{i}^{\prime}\left(e_{\tau} f\left(k_{\tau}\right)\right)=\delta_{i} u_{i}^{\prime}\left(e_{\tau+1} f\left(k_{\tau, \tau+1}\right)\right) e_{\tau+1} f^{\prime}\left(k_{\tau, \tau+1}\right)+ \\
+\delta_{i}^{2} u_{i}^{\prime}\left(e_{\tau+2} f\left(k_{\tau, \tau+2}\right)\right) e_{\tau+2} f^{\prime}\left(k_{\tau, \tau+2}\right)\left(1-e_{\tau+1}\right) f^{\prime}\left(k_{\tau, \tau+1}\right)+\ldots,
\end{gathered}
$$

where we have divided both parts of the equation by $f\left(k_{\tau}\right)>0$.

Consider the left-hand side of equation (32) as a function of $e_{\tau}$ :

$$
\Phi_{\tau}^{i}\left(e_{\tau}\right)=u_{i}^{\prime}\left(e_{\tau} f\left(k_{\tau}\right)\right) .
$$

It is clear that $\Phi_{\tau}^{i}(0)=+\infty$, and

$$
\frac{d \Phi_{\tau}^{i}}{d e_{\tau}}=u_{i}^{\prime \prime}\left(e_{\tau} f\left(k_{\tau}\right)\right) f\left(k_{\tau}\right)<0
$$

Consider also the right-hand side of equation (32) as a function of $e_{\tau}$ :

$$
\begin{gathered}
\Psi_{\tau}^{i}\left(e_{\tau}\right)=\delta_{i} u_{i}^{\prime}\left(e_{\tau+1} f\left(\left(1-e_{\tau}\right) f\left(k_{\tau}\right)\right)\right) e_{\tau+1} f^{\prime}\left(\left(1-e_{\tau}\right) f\left(k_{\tau}\right)\right)+ \\
\delta_{i}^{2} u_{i}^{\prime}\left(e_{\tau+2} f\left(\left(1-e_{\tau+1}\right) f\left(\left(1-e_{\tau}\right) f\left(k_{\tau}\right)\right)\right)\right) \cdot \\
\cdot e_{\tau+2} f^{\prime}\left(\left(1-e_{\tau+1}\right) f\left(\left(1-e_{\tau}\right) f\left(k_{\tau}\right)\right)\right)\left(1-e_{\tau+1}\right) f^{\prime}\left(\left(1-e_{\tau}\right) f\left(k_{\tau}\right)\right)+\ldots
\end{gathered}
$$

Calculate its derivative (divided by $f\left(k_{\tau}\right)>0$ ):

$$
\begin{gathered}
\frac{d \Psi_{\tau}^{i}}{d e_{\tau}}=-\delta_{i} u_{i}^{\prime \prime}\left(e_{\tau+1} f\left(k_{\tau, \tau+1}\right)\right)\left(e_{\tau+1} f^{\prime}\left(k_{\tau, \tau+1}\right)\right)^{2}- \\
\delta_{i} e_{\tau+1} u_{i}^{\prime}\left(e_{\tau+1} f\left(k_{\tau, \tau+1}\right)\right) f^{\prime \prime}\left(k_{\tau, \tau+1}\right)-\ldots- \\
\delta_{i}^{2} u_{i}^{\prime \prime}\left(e_{\tau+2} f\left(k_{\tau, \tau+2}\right)\right)\left(e_{\tau+2} f^{\prime}\left(k_{\tau, \tau+2}\right)\left(1-e_{\tau+1}\right) f^{\prime}\left(k_{\tau, \tau+1}\right)\right)^{2}- \\
\delta_{i}^{2} u_{i}^{\prime}\left(e_{\tau+2} f\left(k_{\tau, \tau+2}\right)\right) e_{\tau+2} f^{\prime \prime}\left(k_{\tau, \tau+2}\right)\left(\left(1-e_{\tau+1}\right) f^{\prime}\left(k_{\tau, \tau+1}\right)\right)^{2}-\ldots- \\
\delta_{i}^{2} u_{i}^{\prime}\left(e_{\tau+2} f\left(k_{\tau, \tau+2}\right)\right) e_{\tau+2} f^{\prime}\left(k_{\tau, \tau+2}\right)\left(1-e_{\tau+1}\right) f^{\prime \prime}\left(k_{\tau, \tau+1}\right)-\ldots
\end{gathered}
$$

Due to the strict concavity of $u_{i}$ and concavity of $f$,

$$
\frac{d \Psi_{\tau}^{i}}{d e_{\tau}}>0
$$


Note that this result holds even when $f(k)$ is linear, i.e., if $f^{\prime \prime}(k)=0$.

Each term in $\Psi_{\tau}^{i}$ contains a multiplier of the form

$$
u_{i}^{\prime}\left(e_{t} f\left(\left(1-e_{t-1}\right) f\left(\left(1-e_{t-2}\right) f\left(\cdots f\left(\left(1-e_{\tau}\right) f\left(k_{\tau}\right)\right)\right)\right)\right)\right) .
$$

Clearly, if $e_{\tau}=1$, then $f(0)=0$, and therefore $\Psi_{\tau}^{i}(1)=+\infty$.

It follows that there exists a unique solution to the equation

$$
\Phi_{\tau}^{i}\left(e_{\tau}\right)=\Psi_{\tau}^{i}\left(e_{\tau}\right)
$$

and this solution lies strictly in $(0,1)$. Thus there is a unique time $\tau$ preferred consumption rate for agent $i$.

Note also that we have

$$
\frac{\partial^{2} V_{\tau}^{i}}{\partial e_{\tau}^{2}}=\frac{d}{d e_{\tau}}\left(\Phi_{\tau}^{i}\left(e_{\tau}\right)-\Psi_{\tau}^{i}\left(e_{\tau}\right)\right)<0,
$$

so the preferences of agent $i$ in voting over the time $\tau$ consumption rate are strictly concave.

Since time $\tau$ and agent $i$ were chosen arbitrarily, this actually implies that the preferences of each agent in voting over every consumption rate are single-peaked.

Proposition 2 immediately follows from Lemma 3. Now let us show that when agents have the same instantaneous utility function and the same expectations, for all $\tau$ (except $\tau=T$ in the finite horizon case) higher values of the discount factor $\delta_{i}$ correspond to lower values of the preferred time $\tau$ consumption rate $e_{\tau}^{i *}$.

Lemma 4. Suppose all agents have the same instantaneous utility function $u(c)$ and the same non-degenerate expectations $E_{\tau+1, T}$. If $\delta_{i}>\delta_{j}$, then $e_{\tau}^{i *}<e_{\tau}^{j *}$.

Proof. It follows from (31) by the implicit function theorem that

$$
\frac{d e_{\tau}^{i *}}{d \delta_{i}}=-\left(\frac{\partial^{2} V_{\tau}^{i}}{\partial e_{\tau} \partial \delta_{i}}\right) /\left(\frac{\partial^{2} V_{\tau}^{i}}{\partial e_{\tau}^{2}}\right)
$$

By Lemma 3, we know that

$$
\frac{\partial^{2} V_{\tau}^{i}}{\partial e_{\tau}^{2}}<0
$$

It is also clear that

$$
\begin{gathered}
\frac{\partial^{2} V_{\tau}^{i}\left(e_{\tau}\right)}{\partial e_{\tau} \partial \delta_{i}}=\frac{\partial}{\partial \delta_{i}}\left(\Phi_{\tau}^{i}\left(e_{\tau}\right)-\Psi_{\tau}^{i}\left(e_{\tau}\right)\right)=-\frac{\partial}{\partial \delta_{i}} \Psi_{\tau}^{i}\left(e_{\tau}\right)= \\
-u^{\prime}\left(e_{\tau+1} f\left(k_{\tau, \tau+1}\right)\right) e_{\tau+1} f^{\prime}\left(k_{\tau, \tau+1}\right)- \\
2 \delta_{i} u^{\prime}\left(e_{\tau+2} f\left(k_{\tau, \tau+2}\right)\right) e_{\tau+2} f^{\prime}\left(k_{\tau, \tau+2}\right)\left(1-e_{\tau+1}\right) f^{\prime}\left(k_{\tau, \tau+1}\right)-\ldots<0 .
\end{gathered}
$$

So finally,

$$
\frac{d e_{\tau}^{i *}}{d \delta_{i}}<0
$$

It follows from the median voter theorem that a time $\tau$ voting equilibrium is the preferred consumption rate for the median agent. 


\section{Proof of Theorem 2}

Let $\tau$ be an arbitrary point in time. Consider how agents vote over the time $\tau$ consumption rate under constant non-degenerate expectations $E_{\tau+1, \infty}=\{e, e, \ldots\}$.

The objective function of agent $i$ in the time $\tau$ voting problem under these expectations is given by:

$$
\begin{gathered}
V_{\tau}^{i}\left(k_{\tau}, e_{\tau}, E_{\tau+1, \infty}\right)=u_{i}\left(e_{\tau} A k_{\tau}\right)+ \\
\delta_{i} u_{i}\left(e\left(1-e_{\tau}\right) A^{2} k_{\tau}\right)+\delta_{i}^{2} u_{i}\left(e(1-e)\left(1-e_{\tau}\right) A^{3} k_{\tau}\right)+\ldots
\end{gathered}
$$

It follows from Lemma 3 that preferences of agent $i$ are concave, and her preferred time $\tau$ consumption rate, $e_{\tau}^{i *}$, is the unique solution to the following equation:

$$
\frac{\partial V_{\tau}^{i}\left(k_{\tau}, e_{\tau}, E_{\tau+1, \infty}\right)}{\partial e_{\tau}}=0
$$

Using the fact that $u_{i}^{\prime}(c)=c^{-\rho_{i}}$, equation (33) takes the form

$$
\begin{aligned}
& A k_{\tau}\left(e_{\tau} A k_{\tau}\right)^{-\rho_{i}}=\delta_{i} e A^{2} k_{\tau}\left(e\left(1-e_{\tau}\right) A^{2} k_{\tau}\right)^{-\rho_{i}}+ \\
& \delta_{i}^{2} e(1-e) A^{3} k_{\tau}\left(e(1-e)\left(1-e_{\tau}\right) A^{3} k_{\tau}\right)^{-\rho_{i}}+\ldots
\end{aligned}
$$

Dividing both parts of the above equation by $\left(A k_{\tau}\right)^{1-\rho_{i}}$, we get

$$
\begin{aligned}
& \left(e_{\tau}\right)^{-\rho_{i}}=\left(1-e_{\tau}\right)^{-\rho_{i}}\left(A \delta_{i} e(A e)^{-\rho_{i}}+\right. \\
& \left.A^{2} \delta i^{2} e(1-e)\left(A^{2} e(1-e)\right)^{-\rho_{i}}+\ldots\right),
\end{aligned}
$$

or

$$
\begin{gathered}
\left(\frac{1-e_{\tau}}{e_{\tau}}\right)^{\rho_{i}}= \\
A \delta_{i}\left(e(A e)^{-\rho_{i}}+A \delta_{i} e(1-e)\left(A^{2} e(1-e)\right)^{-\rho_{i}}+\ldots\right) .
\end{gathered}
$$

It follows from (14) that

$$
\delta_{i}(A(1-e))^{1-\rho_{i}}<1,
$$

so that the infinite series in the right-hand side converges. Therefore,

$$
\begin{gathered}
e(A e)^{-\rho_{i}}+A \delta_{i} e(1-e)\left(A^{2} e(1-e)\right)^{-\rho_{i}}+\ldots= \\
A^{-\rho_{i}}(e)^{1-\rho_{i}}\left(1+\delta_{i}(A(1-e))^{1-\rho_{i}}+\delta_{i}^{2}(A(1-e))^{2\left(1-\rho_{i}\right)}+\ldots\right)= \\
\frac{A^{-\rho_{i}} e^{1-\rho_{i}}}{1-\delta_{i}(A(1-e))^{1-\rho}} .
\end{gathered}
$$

Using (15) and the above equation, we conclude that the preferred time $\tau$ consumption rate for agent $i, e_{\tau}^{i *}$, is a solution to the following equation:

$$
\left(\frac{1-e_{\tau}}{e_{\tau}}\right)^{\rho_{i}}=\frac{\left(\frac{1+\gamma_{i}}{A}\right)^{\rho_{i}} e^{1-\rho_{i}}}{1-\left(\frac{1+\gamma_{i}}{A}\right)^{\rho_{i}}(1-e)^{1-\rho_{i}}} .
$$

Note that the preferred time $\tau$ consumption rate for each agent is independent of the current capital stock $k_{\tau}$, and depends only on constant expectations $e$. 
It can be easily checked that for all $e \neq 0$,

$$
\frac{d e_{\tau}^{i *}}{d \gamma_{i}}<0
$$

It follows that for any constant non-degenerate expectations $e$, the preferred time $\tau$ consumption rates for different agents are naturally ordered by the agents' growth rates:

$$
\gamma_{i}<\gamma_{j} \Rightarrow e_{\tau}^{i *}>e_{\tau}^{j *}
$$

Hence for any time $\tau$ and any constant nonzero expectations $e$, the winner in voting over the time $\tau$ consumption rate is the preferred time $\tau$ consumption rate for the agent with the median growth rate $\gamma_{\text {med }}$.

Thus the balanced-growth voting equilibrium is the preferred time $\tau$ consumption rate for the median agent which coincides with constant expectations. It follows that the balanced-growth voting equilibrium is a solution to (34) for $\gamma_{i}=\gamma_{\text {med }}$, such that $e_{\tau}=e$. It is clear that the balanced-growth voting equilibrium is unique, independent of $k_{0}$, and given by

$$
e^{*}=1-\frac{1}{A}\left(1+\gamma_{m e d}\right)
$$

\section{Proof of Theorem 3}

Consider the constant sequence of consumption rates $E^{*}=\left\{e^{*}, e^{*}, \ldots\right\}$, where $e^{*}$ is given by (18). Consider a fictitious agent with the discount factor $\delta_{\text {med }}$ and instantaneous utility function $u_{i}(c)$, i.e., the agent with the discount factor of the median agent and instantaneous utility function of agent $i$. By the previous reasoning, the sequence $E^{*}$ is the optimum in terms of consumption rates for this agent.

Let $\tau$ be an arbitrary point in time. Suppose that expectations of agents are constant and equal to $E_{\tau+1, \infty}=\left\{e^{*}, e^{*}, \ldots\right\}$. It follows from Lemma 3 that preferences of each agent in voting over the time $\tau$ consumption rate are strictly concave. Consider agent $i$ with the discount factor $\delta_{i}$ and the instantaneous utility function $u_{i}(c)$. She has the unique preferred time $\tau$ consumption rate $e_{\tau}^{i *}$. Lemma 4 allows us to compare $e_{\tau}^{i *}$ with $e^{*}$, which is the preferred time $\tau$ consumption rate for the fictitious agent considered above. It follows from Lemma 4 that if $\delta_{i}<\delta_{\text {med }}$ then $e_{\tau}^{i *}>e^{*}$. At the same time, if $\delta_{i}>\delta_{\text {med }}$ then $e_{\tau}^{i *}<e^{*}$.

Hence the winner in voting over the time $\tau$ consumption rate under constant expectations $E_{\tau+1, \infty}=\left\{e^{*}, e^{*}, \ldots\right\}$ is precisely $e^{*}$ given by (18). Since the point in time $\tau$ is chosen arbitrarily, $e^{*}$ is the winner in voting over each consumption rate under expectations $\left\{e^{*}, e^{*}, \ldots\right\}$. Thus the sequence $\left\{e^{*}, e^{*}, \ldots\right\}$ is an intertemporal voting equilibrium, and hence $e^{*}$ is a steady-state equilibrium.

It remains to show that this steady-state voting equilibrium is unique. Suppose that there is another steady-state voting equilibrium $\tilde{e}$. Consider an arbitrary point in time $\tau$. Suppose that expectations of agents are constant and equal to $E_{\tau+1, \infty}=\tilde{E}=\{\tilde{e}, \tilde{e}, \ldots\}$. It follows from Lemma 3 that preferences of each agent in voting over the time $\tau$ consumption rate are concave. By the median voter theorem, $\tilde{e}$ is the most preferred consumption rate for some "median" voter. Clearly, $\tilde{e}$ is the preferred consumption rate for this same agent for all $\tau=0,1, \ldots$. Therefore, the sequence $\{\tilde{e}, \tilde{e}, \ldots\}$ is a step-by-step intertemporal optimum for this agent. 
Denote the discount factor of this agent by $\tilde{\delta}$. Consider the corresponding $\tilde{k}$, which is the unique positive solution to the equation $k=(1-\tilde{e}) f(k)$. Clearly, $\tilde{k}$ is determined by the "modified golden rule" for this agent: $\tilde{\delta} f^{\prime}(\tilde{k})=1$. It follows that $\tilde{e}$ depends only on $\tilde{\delta}$, and is independent of the instantaneous utility function of this agent. In other words, $\tilde{e}$ is the preferred time $\tau$ consumption rate for the fictitious agent with the discount factor $\tilde{\delta}$ and any instantaneous utility function, in voting over $e_{\tau}$ given $k_{\tau}=\tilde{k}$ and expectations $\tilde{E}$.

Now suppose that $\tilde{e}>e^{*}$, and thus $\tilde{\delta}<\delta_{\text {med }}$. Consider agent $i$ with the discount factor $\delta_{i} \geq \delta_{\text {med }}$ and the instantaneous utility function $u_{i}(c)$. Lemma 4 allows us to compare $e_{\tau}^{i *}$, the preferred time $\tau$ consumption rate for agent $i$, with $\tilde{e}$, the preferred time $\tau$ consumption rate for the fictitious agent with the instantaneous utility function $u_{i}(c)$ and the discount factor $\tilde{\delta}$. Since $\delta_{i}>\tilde{\delta}$, Lemma 4 states that $e_{\tau}^{i *}<\tilde{e}$.

Hence for at least $\frac{N+1}{2}$ agents their preferred time $\tau$ consumption rates are lower then $\tilde{e}$. It follows that $\tilde{e}$ is not a Condorcet winner in voting over the time $\tau$ consumption rate under expectations $\tilde{E}$. Thus $\tilde{e}>e^{*}$ cannot be a steady-state voting equilibrium.

In the case where $\tilde{e}<e^{*}$, the previous argument can be slightly modified. Here for at least $\frac{N+1}{2}$ agents their preferred time $\tau$ consumption rates in voting over the time $\tau$ consumption rate under expectations $\tilde{E}$ are greater than $\tilde{e}$. Thus $\tilde{e}$ is not a Condorcet winner in this voting, and cannot be a steady-state voting equilibrium.

It follows that $e^{*}$ is the unique steady-state voting equilibrium.

\section{References}

Beck, N. (1978). Social Choice and Economic Growth. Public Choice, 33 (2), pp. 33-48.

Becker, R. A. (2006). Equilibrium Dynamics with Many Agents. In Mitra, T., Dana, R.A., Le Van, C., and Nishimura, K., editors, Handbook on Optimal Growth 1. Discrete Time, pp. 385-442. Springer, Berlin Heidelberg.

Bellman, R. (1957). Dynamic Programming. Princeton University Press, Princeton, NJ.

Bernheim, B. D. and Slavov, S. N. (2007). A Solution Concept for Majority Rule in Dynamic Settings. Review of Economic Studies, 76, pp. 33-62.

Borissov, K., Brechet, T., and Lambrecht, S. (2014a). Environmental Policy in a Dynamic Model with Heterogeneous Agents and Voting. In Moser, E., Semmler, W., Tragler, G., and Veliov, V., editors, Dynamic Optimization in Environmental Economics, pp. 37-60. Springer, Berlin.

Borissov, K., Hanna, J., and Lambrecht, S. (2014b). Public Goods, Voting, and Growth. Working Paper Ec-01/14, EUSP Department of Economics.

Borissov, K. and Pakhnin, M. (2014). Economic Growth and Property Rights on Natural Resources. Working Paper CE3S-05/14, Centre for Energy and Environmental Economics Studies.

Boylan, R. T. (1995). Voting over Investment. Journal of Mathematical Economics, 26, pp. 187-208. 
Boylan, R. T., Ledyard, J., and McKelvey, R. D. (1996). Political Competition in a Model of Economic Growth: Some Theoretical Results. Economic Theory, 7, pp. 191-205.

Bucovetsky, S. (1990). Majority Rule in Multi-dimensional Spatial Models. Social Choice and Welfare, 7, pp. 353-368.

Castillo, M., Ferraro, P. J., Jordan, J. L., and Petrie, R. (2011). The Today and Tomorrow of Kids: Time Preferences and Educational Outcomes of Children. Journal of Public Economics, 95 (11-12), pp. 1377-1385.

Davis, O., DeGroot, M., and Hinich, M. (1972). Social Preference Orderings and Majority Rule. Econometrica, 40, pp. 147-157.

De Donder, P., Le Breton, M., and Peluso, E. (2012). Majority Voting in Multidimensional Policy Spaces: Kramer-Shepsle versus Stackelberg. Journal of Public Economic Theory, 14, pp. 879-909.

Ekeland, I. and Scheinkman, J. A. (1986). Transversality Conditions for Some Infinite Horizon Discrete Time Optimization Problems. Mathematics of Operations Research, 11 (2), pp. 216-229.

Gale, D. (1967). On Optimal Development in a Multi-Sector Economy. The Review of Economic Studies, 34 (1), pp. 1-18.

Gollier, C. and Weitzman, M. L. (2010). How should the distant future be discounted when discount rates are uncertain? Economics Letters, 107 (3), pp. 350-353.

Heal, G. and Millner, A. (2014). Resolving intertemporal conflicts: Economics vs. Politics. Working Paper 196, Centre for Climate Change Economics and Policy.

Jackson, M. O. and Yariv, L. (2015). Collective Dynamic Choice: The Necessity of Time Inconsistency. American Economic Journal: Microeconomics, 7 (4), pp. 150-178.

Koopmans, T. C. (1967). Objectives, Constraints and Outcomes in Optimal Growth Models. Econometrica, 35, pp. 1-15.

Kramer, G. H. (1972). Sophisticated Voting over Multidimensional Choice Spaces. Journal of Mathematical Sociology, 2, pp. 165-180.

Kramer, G. H. (1973). On a Class of Equilibrium Conditions for Majority Rule. Econometrica, 41, pp. 285-297.

McKelvey, R. (1976). Intransitivities in Multidimensional Voting Models and Some Implications for Agenda Control. Journal of Economic Theory, 12, pp. 472-482.

Pearce, D., Groom, B., Hepburn, C., and Koundouri, P. (2003). Valuing the future. World Economics, 4 (2), pp. 121-141.

Peleg, B. and Yaari, M. (1973). On the Existence of a Consistent Course of Action when Tastes are Changing. Review of Economic Studies, 40 (3), pp. 391-401.

Phelps, E. S. and Pollak, R. A. (1968). On Second-Best National Saving and Game Equilibrium Growth. The Review of Economic Studies, 35 (2), pp. 185-199. 
Plott, C. (1967). A Notion of Equilibrium and Its Possibility under Majority Rule. American Economic Review, 57, pp. 787-806.

Pollak, R. A. (1968). Consistent Planning. Review of Economic Studies, 2, pp. 201-208.

Ramsey, F. P. (1928). A Mathematical Theory of Saving. Economic Journal, 38, pp. $543-559$.

Schaner, S. G. (2015). Do Opposites Detract? Intrahousehold Preference Heterogeneity and Inefficient Strategic Savings. American Economic Journal: Applied Economics, 7 (2), pp. 135-174.

Shepsle, K. A. (1979). Institutional Arrangements and Equilibrium in Multidimensional Voting Models. American Journal of Political Science, 23, pp. 27-59.

Strotz, R. H. (1955-1956). Myopia and Inconsistency in Dynamic Utility Maximization. Review of Economic Studies, 23 (3), pp. 165-180.

Traeger, C. P. (2013). Discounting under uncertainty: Disentangling the Weitzman and the Gollier effect. Journal of Environmental Economics and Management, 66 (3), pp. $573-582$.

Wang, M., Rieger, M. O., and Hens, T. (2010). How Time Preferences Differ: Evidence from 45 Countries. Research Paper 09-47, Swiss Finance Institute. 


\section{Working Paper Series in Economics}

recent issues

No. 77 Kirill Borissov, Mikhail Pakhnin, Clemens Puppe: On discounting and voting in a simple growth model, December 2015

No. 76 Nikolaus Schweizer and Nora Szech: A quantitative version of Myerson regularity, December 2015

No. 75 Tim Deeken: Schumpeterian growth with technological interdependence: An application to US states, November 2015

No. 74 Tim Deeken: Knowledge spillovers: On the impact of genetic distance and data revisions, November 2015

No. 73 Christian Feige and Karl-Martin Ehrhart: Voting and transfer payments in a threshold public goods game, November 2015

No. 72 Steffen Huck, Nora Szech, Lukas M. Wenner: More effort with less pay: On information avoidance, belief design and performance, September 2015

No. 71 Florian Kreuchauff and Vladimir Korzinov: A patent search strategy based on machine learning for the emerging field of service robotics, August 2015

No. 70 Christian Feige: Success rates in simplified public goods games - a theoretical model, June 2015

No. 69 Markus Fels: Mental accounting, access motives, and overinsurance, May 2015

No. 68 Ingrid Ott and Susanne Soretz: Green attitude and economic growth, May 2015

No. 67 Nikolaus Schweizer and Nora Szech: Revenues and welfare in auctions with information release, April 2015 